\title{
SOME QUESTIONS CONCERNING HRUSHOVSKI'S AMALGAMATION CONSTRUCTIONS
}

\author{
ASSAF HASSON*
}

\section{INTRODUCTION}

In his book on stable groups [Poi87] Poizat writes (with respect to our 1987 understanding of $\omega$-stable fields): "Nous n'avons pas fait de progrès décisif depuis le temps oú nous chassions les aurochs avec une hache de pierre; nous avons seulment acquis une meilleure comprehension de l'enjeu du problem" ${ }^{1 "}$. Sadly, there is no better way to describe the current situation with respect to the problem of classifying strongly minimal sets. Since the refutation of Zilber's Conjecture, Hrushovski's amalgamation construction of new strongly minimal sets, introduced in [Hru93] and [Hru92], stood in the way of any (naive) attempt of classification of their possible pregeometries. Indeed, our mastery of the techniques underlying these constructions is now better than it used to be, but our understanding of the fundamental questions they give rise to can hardly be said to have improved. To the best of my knowledge, there has not been any progress at all with respect to some questions that have already been asked in Hrushovski's original paper. Several survey papers deal with these amalgamation constructions (e.g. [Wag94], [Bal02] and [Poi02]). These papers are concerned mainly with the construction itself - developing axiomatic frameworks in which it can be carried out - and the known examples it gives rise to. The aim of the present paper is threefold, and different:

- Present a setting - given in the language of (standard) geometric stability theory - in which these construction can be understood.

- Show how new structures which have been recently constructed using these methods (most notably [BMPZ05b] and [BHMPW06]) fit into this framework.

- Point out fundamental questions concerning these constructions, that to the best of my knowledge have not been addressed.

A central theme of this paper is that the major gap in our understanding of the scope of these constructions lies in the technically simpler part, usually known as the free construction, whereas the more involved part of the construction, known as the collapse, is fairly well understood.

\footnotetext{
* Supported by the EPSRC grant no. EP C52800X 1.

${ }^{1}$ We have not made any decisive progress since the time we were chasing aurochs with an ax of stone; we have only obtained a better understanding of the scope of the problem.
} 
The first part of this survey is an attempt to explain the latter statement. The starting point is Zilber's treatment of envelopes, introduced as part of his analysis of totally categorical theories ([Zil93]). These tools were subsequently generalized to the context of $\aleph_{0}$-categorical $\aleph_{0}$-stable structures in [CHL85], and in a more developed form to the construction of smooth approximations of Lie coordinatizable structures in [CH03]. In the exposition we will cover the analogies of the collapse with the construction of [CH03] singling out the crucial differences, and how they can be dealt with, using the fusion over a vector space as a worked out example. This choice is not arbitrary. Of all known examples, this is the one (together with the closely related "Red Fields" of [BMPZ05a]) where all the model-theoretic phenomena discussed herein manifest in a non-trivial way.

The problem of classifying the combinatorial geometries obtained through these amalgamation constructions is the focus of the second part of the paper. The thesis I suggest to explore through a series of questions is that the right object to investigate towards an eventual such classification is the local geometry (or rather - the local geometries) of the regular types of infinite rank in the free construction. These questions seem to me to be crucial not only for the possibly too ambitious classification project, but mostly for our understanding of the scope of existing construction techniques and as a guide in our attempts to develop ones that will go beyond them, possibly reaching into genuinely unknown territory. Hopefully, the exposition of the first section will make at least the statement of the problems clearer.

A few words concerning what this survey will not cover are also in place. Although by no means intended for the experts only, some knowledge of the construction techniques this paper is concerned with will not hurt. A reasonably good understanding of any of the many papers on the subject, from Hrushovski's original papers, through the constructions of [Poi99] or [Bal94] and any of the above mentioned survey papers, should suffice to enable the reader to follow the present text. For the benefit of interested readers, who do not have such an acquaintance with these constructions, a quick survey is given in Sections 2.1, 2.2 and 2.3. Due to the large number of ideas involved in the construction, it may seem that Section 2 goes into the fine details of the collapse. However, this is not the case - many nontrivial technical points (mostly the more context dependent ones) are left out. Readers interested in the details are referred to [Has04]. This survey will also not deal with the construction of strictly stable structures in the style of [Hru98]. Not because they are not interesting, but because they do not seem to fall into the model theoretic framework suggested herein, and I do not have anything new to offer on that matter. Finally, since the main concern of this paper is the construction of new structures of finite rank, the many subtle variants giving rise to, e.g. simple theories [Hru97], will not be 
discussed. For these structures it is not even clear how to define what would a collapse (of finite rank) be, let alone produce one ${ }^{2}$.

Lastly, it should be said that no originality claims are made in this paper. Although it may not be obvious from the texts of [Hru93] and [Hru92], one need not be a detective to realize that their relation with the theory of envelopes as developed in [CH03] was clear to Hrushovski and possibly to others as well. Nevertheless, I hope that there is still room for a survey paper that points this out explicitly, and offers a separation of the ideas needed to overcome the technical difficulties in verifying the validity of the axiomatic framework - for individual instances of the general theory - from the ideas needed to produce a genuinely new construction.

Acknowledgment I would like to thank the many brave people who tried to read earlier - even more unreadable - versions of this paper for their helpful comments and suggestions. Special thank are due to Martin Hils for his scrupulous reading of the very first draft and for his many comments which were of great help.

1.1. Some technicalities. We spell out some permanent assumptions, bibliographical remarks and terminology choices. .

Throughout this survey all theories making any appearance will be countable. It is often helpful - though by no means necessary - to assume the theories in question to be given in a relational language.

In what follows we will be working in models of a theory $T_{\infty}$ which we will assume to be the result of a free amalgamation construction (this will be explained in more detail below). For the moment, it is enough to say that $T_{\infty}$ is the theory of the (unique) countable universal model of some (countable) class $\mathcal{A}$ of finite models of a universal theory $T$ in a language $\mathrm{L}$, where universality is defined with respect to a distinguished class of Lembeddings (which will be called strong or self-sufficient embeddings). In many cases a natural expansion of $T_{\infty}$ by definitions can give quantifier elimination (relative to $T$ ). Though in most places this will not matter, in the present survey $T_{\infty}$ will be considered in $L$, which we will call its natural language.

As already mentioned, the fusion over a vector space will serve us as a guiding example throughout the second section. There are two texts dealing with this construction to which we will be referring. The exposition of [HH06a] is closer in spirit to this survey, but does not contain the full result. The text of [BMPZ05b] does contain the full result but uses a totally different exposition, and in some places, the translation to the language used herein may be obscure. For that reason, and for the sake of readability, crucial lemmas from [BMPZ05b] will be given with a precise reference, together

\footnotetext{
${ }^{2}$ It is not the simplicity of the structure that is the problem, but rather the fact that, in general, the 1-dimensional sets in the resulting structures will not be weak linear geometries in the sense of [CH03].
} 
with a "translation" in the text. Nonetheless, the reader may find a handy copy of [BMPZ05b] useful.

Unfortunately, there is no terminology which is generally accepted for dealing with the technicalities of Hrushovski's amalgamation constructions. When dealing with it here, I will adopt - for most purposes - the terminology of [BMPZ05b]. Partly since this will serve as the main example guiding us through the text, but mostly because I like it. As far as geometric stability theory terminology is concerned, less common definitions will be given in the text, but the reader will be assumed to be acquainted with such notions as orthogonality, almost orthogonality, regular types and their local geometries etc. A good source for readers wishing to refresh their memory with these definitions and their basic properties is [Pil96].

\section{From enVelopes to PSEUdo-EnVElopes}

One of the main obstacles for a model theorist trying to understand Hrushovski's amalgamation constructions is that the literature in the subject is given in specialized (and non standard) terminology based on long technical definitions (codes, parasitic, primitive, pre-algebraic, strong and self-sufficient extensions and difference sequences to name just a few). The main aim of this section, is to show that the - usually technically more involved - stage of the construction known as "the collapse" can be understood in standard geometric stability theoretic terms.

Though given in standard terms, the construction involves quite a few subtle techniques, the details of which non-expert readers may find tedious and not easy to follow. For that reason, the first three sub-sections are intended to give a self-contained general overview of the ideas appearing in the construction. These should hopefully give sufficient background for readers not interested in the fine details of the construction and wishing to skip directly to Section 3, and a smooth introduction to the more technical parts for the others.

2.1. The free construction. In many texts concerning Hrushovski's amalgamation technique it is pointed out that the construction is carried out in two different steps: first a free amalgamation construction, which usually produces a structure of infinite rank, and then a collapse stage which produces one of finite rank. In practice, however, either the first stage is skipped altogether or the collapse looks more like a rehash (albeit with additional difficulties) of the free amalgamation stage, taking place in a more restrictive (universal) class. As such, the collapse may be seen more like an independent construction than a second stage necessarily following the free amalgamation. One of the aims of this section is to make clear the role of each of the two stages in the construction.

From the technical point of view, the free amalgamation construction (free construction, for short) is rather straightforward and seems to be quite well 
understood. What may be slightly less clear is that it provides the playground where all the rest of the work will be done. More importantly, if we view the construction of combinatorial geometries with prescribed properties as the ultimate goal ${ }^{3}$, this is the stage where this geometry is obtained (and later slightly modified to fit into a structure of finite rank). Zilber (e.g. in [Zil05]) interprets this construction - or rather its complete axiomatization - as one characterized by:

- A 'Generalized Schanuel' condition requiring that some pre-determined integer valued pre-dimension function $\delta$, defined on the set of finite models of a universal theory, $T^{\forall}$, be non-negative (informally, Zilber describes this condition as asserting that "no over-determined system of equations has a solution").

- An existential-closedness condition (with respect to the universal theory determined by the Schanuel condition), which can be interpreted informally as "every systems of equations which is not overdetermined has a solution".

Reversing Zilber's point of view, the pre-dimension function, $\delta$, can be thought of as suggesting, given a (finite) set of "varieties" in $n$-space, what should be the transcendence degree of a generic point in their intersection or, in other words, what is the dimension of their intersection.

For example, in the case of fusion of two strongly minimal theories $T_{1} ; T_{2}$ (with quantifier elimination) over a totally categorical $T_{0}=T_{1} \cap T_{2}$, for a finite $A=T_{1}^{\forall} \cup T_{2}^{\forall}$ the pre-dimension function is given by:

$$
\delta(A)=\mathrm{MR}_{1}(\operatorname{tp}(A))+\mathrm{MR}_{2}(\operatorname{tp}(A))-\mathrm{MR}_{0}(\operatorname{tp}(A))
$$

where $\mathrm{MR}_{i}$ denotes Morley rank in the sense of $T_{i}$ (in other words, $\mathrm{MR}_{i}$ is the dimension of the $T_{i}$-locus of $A$, and $\delta(A)$ is the "right" dimension of the intersection of these loci). So the Schanuel condition simply says that $\delta(A) \geq 0$ for all finite $A$, i.e. that there are no solutions to equations, which on dimension theoretical grounds should not have one.

The existential-closedness condition says that this is the only reason for a system of equations not to have a solution. To give a more accurate description of this axiom denote $L:=L\left(T^{\forall}\right.$ ) (for $T^{\forall}$ the universal theory appearing in the axiomatization). We define a class of distinguished "strong" (or self-sufficient) embeddings $f: A \hookrightarrow B$ for $A, B \models T^{\forall}$, by requiring that

$$
\delta(f(A))=\min \left\{\delta\left(A^{\prime}\right): f(A) \sqsubseteq A^{\prime} \sqsubseteq B\right\}^{\dagger} .
$$

\footnotetext{
${ }^{3}$ It may be interesting to know whether a bad group of rank 3 can be characterized in terms of the associated combinatorial geometry. But even if this is impossible, already the construction of a non-CM-trivial geometry, admitting a group configuration but not a field configuration will be a big step forward.

${ }^{\dagger}$ In most cases there is no harm taking $\sqsubseteq$ to coincide with $\subseteq$. This is not true in general, and the definition of $\sqsubseteq$ has to (and can) be made precise axiomatically, but we will not need it. For the present discussion suffice it if we say that in the case of fusion over a vector space it coincides with "sub-vector space".
} 
If $A \subseteq B$ we will write $A \leq B$ (and say that $A$ is strong in $B$ ) if the identity is a strong embedding of $A$ into $B$. In those terms 'Schanuel condition can be stated as $\emptyset \leq A$ for all $A$. We will denote $\mathcal{C}_{0}:=\left\{\emptyset \leq A=T_{\infty}\right\}$. The existential-closedness axiom scheme can now be stated as the requirement that for every model $M$ and $A \subseteq M$, if $A \leq B \in \mathcal{C}_{0}$ (finite) then there exists a strong embedding of $B$ into $M$ over $A$.

We leave it as an exercise to the reader to verify that in the case of the fusion over a (totally categorical) vector space both axiom schemes are first order axiomatizable. From now on we will denote the above axiom schemes by $T_{\infty}$ and always assume that they are elementary. Naturally, the first step in the process is to show that $T_{\infty}$ is consistent. This is done by proving that the class $\left(\mathcal{C}_{0}, \leq\right)$ has the amalgamation property and that $\leq$ is transitive on $\mathcal{C}_{0}^{\ddagger}$ (so it admits a Fraïssé limit, which is easily verified to be a model of $\left.T_{\infty}\right)$.

2.2. The geometric structure of $T_{\infty}$. As mentioned in the previous subsection, $T_{\infty}$ provides us with the setting in which the collapse will eventually take place. In this sub-section we describe the features of this setting.

A key observation towards that end (see, e.g. Lemma 6.2 of [HH06a] for the fusion over a vector space) is that (if $\delta$ is chosen carefully enough) for any $A \subseteq B=T^{\forall}$, if $\emptyset \leq B$, there exists a unique minimal $A \subseteq C \leq B$ which we will denote $\operatorname{cl}_{B}(A)$ (and call the self-sufficient closure of $A$ in $B$ - omitting $B$, if the context is clear). From now on we will assume that $\operatorname{cl}(A) \subseteq \operatorname{acl}_{T_{\infty}}(A)$ for all $A$. We will see below that in structures where this is not the case, it is not clear how to define the collapse, and since this is the main concern of this section, this assumption is natural. It may also be worth noting that if for every finite $A$ there exists a finite $B$ such that $A \sqsubseteq B$ then the above assumption will always hold. For a discussion of a construction where this is not the case, see [Poi01] (where in fact the self-sufficient closure is not well defined).

Under the assumption that $\mathrm{cl}$ is well-defined and algebraic it is not hard to check that $\operatorname{cl}(B a) \cong \operatorname{cl}(A a) \oplus_{A} \operatorname{cl}(B A)$ is a notion of independence (where $B \oplus_{A} C$ denotes the free amalgam of $B$ with $C$ over $A$ ). It follows that $T_{\infty}$ is stable and this notion of independence must coincide with non-forking ${ }^{4}$. So we get, in fact, that $T_{\infty}$ is superstable, and in order not to overload the exposition, we will assume (as is usually the case) that there are enough formulae in $T_{\infty}$ to isolate each type in its $U$-rank, implying that $T_{\infty}$ is $\omega$-stable and $U$-rank is equal to Morley rank.

Consider a set $A=\operatorname{acl}_{T_{\infty}}(A) \subseteq M \models T_{\infty}$ and $\bar{b}$ such that:

$$
\text { - } \delta(\bar{b} / A):=\min \left\{\delta\left(A^{\prime} \bar{b}\right)-\delta\left(A^{\prime}\right): A^{\prime} \subseteq A \text {, finite }\right\}=0
$$

\footnotetext{
${ }^{\ddagger}$ In most cases $A \leq B$ will be equivalent to $\delta(X \cap A) \leq \delta(X)$ for all $X \sqsubseteq B$. In cases where this is not the case, the latter, stronger condition, may be the right definition of $A \leq B$

${ }^{4}$ This will only be true under the additional assumption that if $A \leq B_{1}, B_{2}$ then the isomorphism type of the free amalgam is uniquely determined.
} 
- $\delta\left(\bar{b}^{\prime} / A\right)>0$ for all $\bar{b}^{\prime} D \subseteq \bar{b}$.

By our characterization of forking and the minimality of $\bar{b}$ we get that $\operatorname{tp}(\bar{b} / A)$ is minimal, and by our assumption it must be strongly minimal. Call such a type simply pre-algebraic. More generally, call $\bar{b}$ such that $\delta(\bar{b} / A)=0$ pre-algebraic, and $\delta$-minimal if $\delta\left(\bar{b}^{\prime} / A\right)>\delta(\bar{b} / A)$ for all $\overline{b^{\prime}} \subseteq \bar{b}$. Naturally, if $A \leq B$ and $B \backslash A$ is finite (more precisely, if there exists a finite set $\bar{b} \subseteq B$ such that $\operatorname{cl}(A \bar{b})=B$ ) there exists a decomposition (essentially unique) of $\operatorname{tp}(B / A)$ into $\delta$-minimal types. We conclude, that the $\delta$-minimal types coordinatize $T_{\infty}$. That is, for every $A \leq B$ as above there are $\bar{b}_{1}, \ldots, \bar{b}_{2} \subseteq \bar{b}$ such that $\operatorname{acl}\left(A \bar{b}_{1}, \ldots, \bar{b}_{n}\right)=\operatorname{acl}(A \bar{b})$ and $\operatorname{tp}\left(\bar{b}_{i} / \operatorname{acl}\left(A \bar{b}_{1}, \ldots, \bar{b}_{i-1}\right)\right)$ is $\delta$-minimal for all $1 \leq i \leq n$. This fact is crucial in everything that follows, but in many cases it is so obvious that it is in fact not mentioned explicitly. In the fusion over a vector space this is essentially the content of Proposition 4.7 of [HH06a].

To simplify the exposition further, we will assume the pre-algebraic types are dense in $T_{\infty}$ and that up to non-orthogonality $T_{\infty}$ has one ${ }^{5}$ type $p_{\omega}$ of rank $\omega$, which we will call the generic type of $T_{\infty}$. Note that e.g. in bi-colored fields, there are other natural candidates for the title and the unique regular type of rank $\omega$ is usually called the "colored generic". This suggests that a more appropriate name for $p_{\omega}$ might be "the generic regular type of $T_{\infty}$ ", but for the sake of clarity, we will use the shorter terminology. Collecting all of the above together, it is now easy to check that the simply pre-algebraic types together with $p_{\omega}$ form a complete set of representatives of the nonorthogonality classes of regular types in $T_{\infty}$. We will see that the geometries of the regular types have a crucial role in our analysis. In particular, the geometry we set to construct is (essentially) the local geometry of $p_{\omega}$ - given by cl.

At this stage, we can already give a rough idea of the ideology behind the collapse: given $M \models T_{\infty}$ we would like to find a homogeneous, infinite $N \leq M$ such that $\operatorname{dim}_{N}(p)<\infty$ for every strongly minimal type in $S(M)$ based over $N$. The idea is that, if everything works out properly, $N$ will inherit the coordinatization from $M$ (by the homogeneity) and therefore will be $\omega$-stable and unidimensional (because $N$ is infinite it must have some non-algebraic type, and the requirement $\operatorname{dim}_{N}(p)<\infty$ should make all the strongly minimal type of $T_{\infty}$ algebraic, so - by coordinatization - $p_{\omega}$ will be the only non-algebraic type). This would make $\operatorname{Th}(N)$ uncountably categorical, and $p_{\omega}(N)$ it's unique strongly minimal set, as desired.

One way of avoiding trivial solutions to the above project can be to require that if $p \in S^{T_{\infty}}(N)$ is realized in $N$ then $p \perp p_{\omega}$ (this will be slightly elaborated in the closing paragraphs of this sub-section). This would imply that $N$ is not a saturated model of its theory, but the homogeneity will be good enough a replacement.

\footnotetext{
${ }^{5}$ This will not, in general, be the case. However, as long as the strongly minimal types are dense in $T_{\infty}$ the construction is practically unaltered.
} 
It is now clear, that if we want any chance of having both any $M \models \operatorname{Th}(N)$ to inherit the coordinatization from $T_{\infty}$ and $\operatorname{Th}(N)$ to be uncountably categorical, we must require that any strongly minimal $p \in S(N)$ which is realized in some $N \prec_{T_{\infty}} M$ be locally finite. Towards this end, we will have to prove something in the spirit of:

Lemma 2.1. Every strongly minimal set in $T_{\infty}$ is locally finite. In particular, it is locally modular.

The lemma is not true in all free constructions, but if some $p \in S(M)$ were not locally finite, in order for $N=\operatorname{acl}_{T_{\infty}}(N)$ to be unidimensional, we would have to require that $\operatorname{dim}_{N}(p)=0$ (the alternative solution - requiring that $p$ be the unique regular type of $T h(N)$ - is uninteresting). For such a requirement to be consistent delicate questions concerning definability of non-orthogonality to $p$ will have to be addressed ${ }^{6}$.

It is worth noting, however, that even in the presence of non-locally modular strongly minimal types in $T_{\infty}$ it may well be that a collapse of the locally modular types is still possible, resulting in potentially interesting new structures. A good test case where this approach seems possible to implement can be found in [Kir06] where $T_{\infty}$ is interpreted in a differentially closed field, with some of its strongly minimal sets non-orthogonal (in $T_{\infty}$ ) to the field of constants. In this example, there is only one 'problematic' non-orthogonality class, so that the resulting collapsed structure will still be of rank $\omega$, but will not be multidimensional. Similar ideas were already used in the constructions of [HI03].

The importance of the local modularity of the strongly minimal types of $T_{\infty}$ will be discussed in more detail in the next subsection.

Despite of being absolutely crucial for the construction, Lemma 2.1 is not stated explicitly in most texts dealing with the collapse. In the context of the fusion over a vector space the lemma is proved in Proposition 6.8 of [HH06a]. In [BMPZ05b] local finiteness follows from Lemma 6.1 (which we slightly reformulate):

Lemma 2.2. Let $M \models T_{\infty}$ and $N=\operatorname{acl}_{T_{\infty}}(N) \subseteq M$ be such that $\operatorname{dim}_{N}(p)$ is finite for every pre-algebraic type $p \in S(N)$, then any strongly minimal formula $\varphi \in L(N)$ has finitely many solutions in $N$.

However, local modularity only acts behind the scenes of the text in [BMPZ05b]. In [BHMPW06] it is even harder to track down these facts. The somewhat harder proof of local finiteness of strongly minimal sets is rather well hidden in the proof of Lemma 7.3, but is never stated explicitly (though it follows implicitly from the fact that the class $K_{\mu}$ has the amalgamation property).

\footnotetext{
${ }^{6}$ As long as $p$ is locally modular, it is quite hopeful that such questions could be addressed - as was conjectured in [Poi01]. But in the non-locally modular case the situation is much more delicate.
} 
2.3. An overview of the collapse. The ideology underlying the exposition is that once $T_{\infty}$ has been constructed, given $M \models T_{\infty}$ the collapse can be described as obtaining (elementary) means of constructing subsets $N \leq M$, in which the dimensions of simply pre-algebraic types are well controlled (and in particular finite).

To better understand this, remark that our assumption that the prealgebraic types are dense in $T_{\infty}$ would, in most cases, imply that for any type $p \in S^{T_{\infty}}(A)$ and $a=p \mid A$ if $d(a / A)>0$ then $\operatorname{MR}(p)$ is infinite. Since, up to non-orthogonality, $p_{\omega}$ is the unique regular type of infinite rank, it follows that any forking extension of $p_{\omega}$ is domination equivalent to a pre-algebraic type. Since pre algebraic types are totally categorical, any definable subset of $M$ of finite rank has at most finitely many solutions in $N$ (because the simply pre-algebraic ones are finite in $N$ ). In particular, any forking extension of $p_{\omega}$ will be algebraic in $N$, so $p_{\omega}$ - interpreted in $N$ - will be a minimal type.

Such control over the behavior of strongly minimal sets made its first appearance in the totally categorical case, where this process is precisely Zilber's construction of envelopes. Generalized later to the context of $\aleph_{0^{-}}$ categorical, $\aleph_{0}$-stable structures in [CHL85] it reached its ultimate form in [CH03], as part of the characterization of smoothly approximable structures. In many ways, as will be made clear in this section, the collapse can be viewed as a generalization of these constructions. The geometrical ideas underlying the collapse appear already in these early works, and the main technical difficulty in their adaptation to the present context lies in its being out of the $\aleph_{0}$-categorical realm.

Roughly, the main steps in the collapse are:

(1) Prove that the free amalgam is coordinatized by (usually) a unique regular type of (in most cases) infinite rank and families of totally categorical strongly minimal sets.

(2) Identify a good set of representatives of the non-orthogonality classes of strongly minimal sets and collect them in a "standard system" $\mathcal{C}$ where each class is represented (essentially) once.

(3) Show that for a function $\mu: \mathcal{C} \rightarrow \mathbb{N}, \mu$-envelopes of $\emptyset$ (with respect to $\mathcal{C}$ ), i.e. maximal algebraically closed subsets $X \subseteq M$ satisfying $\operatorname{dim}_{X} J \leq \mu(J)$ for all $J \in \mathcal{C}$, are homogeneous (and in particular that, for some $\mu$, they exist).

(4) Find a good elementary analogue of envelopes for which the same construction works.

The coordinatization lemma which forms the first part of the process described above, is usually straight forward and was dealt with in the previous sub-sections, as well as the somewhat trickier and crucial fact that all strongly minimal sets in $T_{\infty}$ are totally categorical.

To obtain a consistent set of conditions of the form "the dimension of $J_{a}$ is at most $\mu\left(J_{a}\right)$ " for a strongly minimal geometry $J_{a}$ (definable in $M$ ), it is most convenient to make sure that such a condition is given only once for 
each non-orthogonality class of geometries. It is therefore important to be able to collect a (non redundant) set of representatives for those classes. The technical term for such collections is systems of standard geometries. This is taken care of in step two in the process described above. The key to the construction of such systems of geometries is definability of orthogonality in $T_{\infty}$. This requires a good understanding of forking and algebraic closure in the free amalgam, but since - as we have already seen - this geometry has an explicit description in the same terms used for the construction of the amalgam it should not, in general, be hard to obtain. Sub-section 2.5 covers these question in some detail.

The construction of envelopes can now follow, almost word by word, the one in $\S 3$ of [CH03]. In this construction, as in the $\aleph_{0}$-categorical case, local modularity of the strongly minimal geometries in question plays an important role by supplying us with the "uniqueness of parallel lines", assuring the homogeneity of envelopes. To better understand this, assume that $X=\operatorname{acl}_{T_{\infty}}(X) \subseteq M=T_{\infty}$ is such that $\operatorname{dim}_{X} p=\mu(p)$ for some $p$, the generic type of a projective space (over a finite field) definable over $X$. If we want to have any chance of $X$ being homogeneous, we must decide given $A$, an affine space of $p$ defined over $X$ - whether $A$ has a point in $X$ or not. The "uniqueness of parallel lines" assures, that if $X=\operatorname{acl}_{T_{\infty}}(X)$ is contained in a $\mu$-envelope and does not contain a point of $A$ then for any $a \in A, X a$ is contained in a $\mu$-envelope too (so the answer is "every affine space defined over $X$ has a point"). In sub-section 2.5 we will see that this is, essentially, all we have to require to assure the homogeneity of envelopes.

But, in general, envelopes will not be elementary. Given a family $J$ of strongly minimal sets, the statement $\operatorname{dim} J_{a}=n$ will not be first order (the reason being that despite the total categoricity of $J_{a}$ we cannot uniformly bound $\left.\left|\operatorname{acl}_{T_{\infty}}(a) \cap J_{a}\right|\right)$. This calls for a first order approximation of $\operatorname{dim} J_{a}=$ $n$, which in [BMPZ05b] was appropriately called pseudo Morley sequence of length $n$. This leads naturally to the concept of pseudo envelopes, which are introduced in section 2.6.

Since pseudo Morley sequences approximate actual Morley sequences, the construction of pseudo envelopes is not very far from the construction of envelopes, with one important exception. Strongly minimal sets in $T_{\infty}$ are totally orthogonal, i.e. if $E_{0}=\operatorname{acl} T_{\infty}\left(E_{0}\right)$ embeds into a $\mu$-envelope, and $a / E_{0}$ is strongly minimal, then for any projective geometry $I$ definable over $E_{a}:=\operatorname{acl}_{T_{\infty}}\left(E_{0} a\right)$ we have $\operatorname{dim}_{E a} I>0$ only if $I \not \perp E_{0}$. This is, of course, a very handy tool in the construction of envelopes. As in the smoothly approximable case, this property follows inductively from the coordinatization of $T_{\infty}$, with the base case following from the fact that if $c=C b\left(\operatorname{tp}\left(a / E_{0}\right)\right)$ then $\operatorname{dim}_{\operatorname{acl}(c)} \operatorname{tp}\left(a / E_{0}\right)=0$. This last equality is not quite true of pseudo Morley sequences, for which an appropriate correction must be proved. The strategy for overtaking this obstruction are discussed in Sub-section 2.7.

Choosing $\mu: \mathcal{J} \rightarrow \mathbb{N}$ for which this construction can be carried out, will assure that no strongly minimal set $I$ definable over a $\mu$-pseudo envelope $E$ 
has infinite dimension in $E$ and by total categoricity (of strongly minimal sets in $\left.T_{\infty}\right)$ in fact $|E \cap I|<\infty$. Homogeneity of pseudo envelopes assures quantifier elimination (modulo $T_{\infty}$ ) so $E$ inherits the coordinatization from $T_{\infty}$. This implies that $E$ is unidimensional, and from the coordinatization, $\omega$-stable. Hence $E$ is uncountably categorical.

We now proceed to describe this construction in more detail. We will use the construction of the fusion over vector spaces as a main example, stressing the translation of the specialized terms of the construction into the standard geometric terminology.

2.4. Envelopes. We make a slight digression to discuss the notion of envelopes, as it was developed in the $\aleph_{0}$-categorical context.

A key step in Zilber's proof of the non-finite axiomatizability of totally categorical theories was noting the importance of envelopes. Defining:

Definition 2.3. Let $T$ be stable, $\mathcal{J}:=\left(\varphi_{1}, \ldots, \varphi_{k}\right)$ a set of pairwise orthogonal strongly minimal sets definable over $\emptyset$ and $X \subseteq M \models T$ any set. A $\mathcal{J}$-envelope $E_{\mathcal{J}}(X)$ of $X$ is a maximal set such that $E_{\mathcal{J}}(X) \downarrow_{X} \mathcal{J}$.

Zilber then proves:

Theorem 2.4. Let $\varphi$ be a strongly minimal set definable in a totally categorical theory $T$ then:

(1) $E_{\varphi}(X)$ is finite for all finite $X$.

(2) For any $m \in \mathbb{N}$ there exists a natural number $s_{m}$ such that for all $X, E_{\varphi}(X)$ is m-elementary (i.e. for all $\bar{a} \in X,|\bar{a}|=m, E_{\varphi}(X) \models$ $\exists \bar{x} \psi(\bar{x}, \bar{a})$ iff $\models \exists \bar{x} \psi(\bar{x}, \bar{a}))$ provided that $|\operatorname{acl}(X) \cap \varphi|>s_{m}$.

(3) $\varphi$-envelopes are homogeneous.

To obtain a possibly better understanding of the nature of envelopes, note that Zilber's theorem proves that if $M$ is the unique countable model of a totally categorical structure, then it is smoothly approximable by envelopes. This means not only that $\operatorname{Th}(M)$ is the limit theory of its envelopes but also that $\operatorname{Aut}(M)$ is the limit (in the natural sense of pointwise convergence) of the groups of automorphisms of envelopes.

Zilber's use of envelopes was subsequently generalized to the context of $\aleph_{0^{-}}$ categorical $\aleph_{0}$-stable structures. In both cases the notion of coordinatization played a crucial role. Recall that (say, in an $\aleph_{0}$-categorical $\aleph_{0}$-stable theory T)

Definition 2.5. If $p \in S(\emptyset)$ is non-algebraic and is $\emptyset$-definable then $p$ coordinatizes $\psi$ if $\operatorname{acl}(x) \cap p \neq \emptyset$ for all $x \in \psi$.

The reader unfamiliar with this notion of coordinatization should have in mind the example of a definable set $\psi(x)$ of rank $n$ and $\{\varphi(x, y): y \in p\}$ an infinite definable normal family of rank $n-1$ subsets of $\psi(x)$. Then by local modularity $\operatorname{MR}(p)=1$ and the $p$-coordinate of $a \in \psi$ is $b=p$ such that $a \in \varphi(x, b)$. 
The main ingredients in Zilber's proof, appearing later also in [CHL85] (in the absence of $\emptyset$-definable equivalence relations) were:

- The theory is coordinatized by a (resp. finitely many) $\aleph_{0}$-categorical strongly minimal set(s).

- $\aleph_{0}$-categorical strongly minimal sets are locally modular.

- If $\psi$ is a non-trivial, $\aleph_{0}$-categorical and non-modular strongly minimal set defined over $\emptyset$ there are an $\emptyset$-definable equivalence relation with finite classes $F$ and a $\emptyset$-definable vector space $V$ such that $(\psi / F, V)$ is an affine space.

- Wrapping all of the above together, if $\mathcal{J}$ is a set of modular coordinatizing geometries, and $T$ is primitive (i.e. does not have nontrivial $\emptyset$-definable equivalence relations) then $E$ is a $\mathcal{J}$-envelope of $X=\operatorname{acl}(X)$ iff $E=\operatorname{acl}(E) \supseteq X$, every $\mathcal{J}$-affine space definable over $E$ has a point in $E$ and $\operatorname{dim}_{E}(J)=\operatorname{dim}_{X}(J)$ for all $J \in \mathcal{J}$.

Ideologically, it should be clear that the homogeneity of envelopes (which plays a crucial role in the proof) must come from their maximality. However it should also be clear that maximality alone is not enough: envelopes have to be algebraically closed, but they also have to include realizations of types (nonorthogonal but) almost orthogonal to $\mathcal{J}$ - as many as possible without increasing the $\mathcal{J}$-dimension. However, in general, given (a finite) $X=\operatorname{acl}(X)$ the order in which we add to $X$ realizations of types $p \perp_{X}^{a} J$ (but non-orthogonal to $J$ ) is not immaterial. In the above setting, the additional ingredient, coming easily from local modularity, is the so called "uniqueness of parallel lines": for strongly minimal types $p_{1} \not \perp p_{2}$ either $p_{1} \perp^{a} p_{2}^{\otimes n}$ for all $n$ or $p_{2} \perp^{a} p_{1}^{\otimes n}$ for all $n$, provided that $p_{1} \perp^{a} p_{2}$.

The construction of envelopes in the works of Zilber and Cherlin-HarringtonLachlan was relatively easy (modulo the hard classification of locally finite pregeometries) due to the fact it used the simple definition of envelopes given above, where we only care about finitely many coordinatizing geometries (based on $\emptyset$ ). In [CH03] the notion of Lie coordinatizable structures was introduced, refining the definition of coordinatization and allowing definable (multidimensional) families of geometries, giving the structure a form of a tree (historically, in the stable case in which we are interested, a related definition of quasi-coordinatizable structures appears already in [CL86] with a close relative in [Lac84], but the one in [CH03] is more accessible, and generalizes easily to the contexts we are interested in):

Definition 2.6. A stable structure $M$ is coordinatized by Lie geometries if it carries a tree structure of finite height with a unique, $\emptyset$-definable root, such that for each $a \in M$ above the root either a is algebraic over its immediate predecessor in the tree ordering, or there exists $b<a$ and $a b$-definable projective geometry $J_{b}$ fully embedded in $M$ and either:

(1) $a \in J_{b}$; or 
(2) there is $c \in M$ with $b<c<a$, and a c-definable affine geometry $\left(J_{c} ; A_{c}\right)$ with vector part $J_{c}$, such that $a \in A_{c}$ and the projectivization of $J_{c}$ is $J_{b}$.

We refer to $\S 2$ of [CH03] for more details concerning the above definition (where it is given in the general, non-stable, context).

Remark 2.7. (1) It may well be that in the stable context the term "coordinatization by linear geometries" is more appropriate than the one given.

(2) For totally categorical theories the two definitions of coordinatization coincide.

(3) For an $\aleph_{0}$-categorical, $\aleph_{0}$-stable theory $T$ the new definition is more subtle. For simplicity, assume that $M=T$ is coordinatizable (in the sense of 2.5) by one strongly minimal type. The coordinatization induces an equivalence relation $\sim$ on $M$. As every $\sim$-equivalence class is itself $\aleph_{0}$-categorical, $\aleph_{0}$-stable, and of lower rank than $\operatorname{MR}(M)$, it is now easy to see how to obtain a tree of coordinatizing geometries on $M$. Indeed, if $M$ is primitive, i.e. admits no non-trivial $\emptyset$;-definable equivalence relations then the definitions coincide.

Gathering the results form Sub-section 2.2, the coordinatization of $T_{\infty}$ by $\delta$-minimal types and the modularity of the strongly minimal ones among them (Lemma 2.1) we start getting the impression that the structures we are interested in can be viewed as a fairly natural, somewhat overgrown, version of (stable) Lie coordinatizable structures.

There are, however, two important differences:

(1) The depth of the graph ${ }^{7}$ of coordinatizing geometries is not - as a rule - finite; and

(2) The generic type $p_{\omega}$ is not, in general, projective.

In the collapse of $T_{\infty}$ the presence of the non-projective generic type can be - for all practical purposes - ignored. Neglecting $p_{\omega}$ causes no harm because, in some sense, it is a grown up type that can take care of itself: $p_{\omega}$ is approximated by types of finite rank (and usually even by strongly minimal types); if we handle the strongly minimal types well enough, compactness will take care of $p_{\omega}$. In fact, if instead of working in a saturated model of $T_{\infty}$ we work in a large existentially closed $\bar{M}=T_{\infty}$, the generic type will not appear in the coordinatizing tree of $\bar{M}^{\S}$.

As for the fact that the tree of (projective) coordinatizing geometries is infinite - in itself it does not cause a serious problem. It is, however, a witness to the fact that $T_{\infty}$ is not $\aleph_{0}$-categorical, which is the main additional

\footnotetext{
${ }^{7}$ Indeed, in general this graph will not be a tree, but it is nonetheless easily stratified, which is all we will need.

${ }^{\S}$ Recall that $T_{\infty}$ is given in its natural language. In particular, if $\bar{a} \leq M, 0<\delta(\bar{a})$ there exists $\bar{a}^{\prime} \models q f \operatorname{tp}(\bar{a})$ with $\bar{a}^{\prime} \not \leq M$. Hence $M$ is existentially closed, iff $\delta(\bar{a})=0$ for all ăleM
} 
difficulty with respect to Lie coordinatizable structures. Instead of working with complete types, we will have to content ourselves with approximating formulas which will require harder work in order to obtain uniformity results needed to make our strategy work.

2.5. Systems of standard geometries. We will now proceed slightly deeper into the description of the strategy of the collapse. We will be working in an $\omega$-stable theory $T_{\infty}$ coordinatized by locally finite strongly minimal types and a unique generic type $p_{\omega}$ of rank $\omega$. Our goal is to construct a homogeneous infinite $N=\operatorname{acl}_{T_{\infty}}(N) \subseteq M$ in which every strongly minimal type $p \in S(N)$ has at most finitely many realizations. As we have already seen, in this setting it will be enough to control the behavior of simply pre-algebraic types.

In order to obtain such control we will adapt a tool form [CH03]. The idea of [CH03] (appearing already in earlier works of Lachlan) is to (definably) collect representatives of non-orthogonality classes of rank 1 types in systems of standard geometries. A standard geometry is, basically, a definable family whose domain is a complete type and whose range is a set of pairwise orthogonal rank 1 projective geometries. A system of standard geometries is a collection of standard geometries where each non-orthogonality class is uniquely represented. In the present context, since $T_{\infty}$ is not $\aleph_{0}$-categorical, things are more complicated, and we will have to extract (and preserve) explicitly much of the information that comes for free in standard systems of geometries. The resulting objects are known, maybe not very informatively, as codes (2-codes in [Hru92]).

In this sub-section we will discuss the guidelines in the construction of codes, avoiding the unpleasant technical details and definitions. The first thing to observe is that if $p_{1}, p_{2} \in S(B)$ are strongly minimal and $p_{1} \not \perp p_{2}$ then there are two options:

- $p_{1}, p_{2}$ are both modular so $p_{1} \not \not_{B}^{a} p_{2}$ with a $B$-definable finite-tofinite correspondence, $f$ between them. If $p_{1}, p_{2}$ are strictly minimal, then $f$ is bijective.

- $p_{1}$ or $p_{2}$ are affine, i.e. locally modular non-modular, in which case their projectivization is based on $B$, reducing the problem to the case where $p_{1}$ is affine and $p_{2}$ projective. In that case $p_{1}$ does not have a solution in $B$ and by the uniqueness of parallel lines, $\operatorname{dim}_{\mathrm{acl}(B a)} p_{2}=$ $\operatorname{dim}_{\operatorname{acl}(B)} p_{2}$ for any $\left.a \models p_{1}\right|_{B}$.

This leads to the following observations:

(1) If we want to control the dimension of $p \in S(N)$ in $N$ it suffices to control the dimension in $N$ of any representative of the nonorthogonality class of $p$.

(2) The representatives of the non-orthogonality classes of strongly minimal types in $T_{\infty}$ which are easier to handle are modular, (preferably even strictly minimal). 
(3) Since, ultimately, we want to be able to axiomatize $\operatorname{Th}(N)$ the choice of representatives should be done in a uniform way.

Which raises, naturally, the question of definability of orthogonality in $T_{\infty}$. Recall that:

Definition 2.8. Let $p \in S(B)$ be a stationary type. We say that orthogonality to $p:=p_{a}$ (for some finite a such that $C b(p) \subseteq \mathrm{dcl}(a)$ ) is definable if for every definable family $R(\bar{x}, \bar{y})$ there exists a formula $O_{a}(\bar{y})$ such that

$$
O_{a}(\bar{y})=\{b: R(\bar{x}, \bar{b}) \perp p\}
$$

The definition of orthogonality is uniform if there exists $\theta \in \operatorname{tp}(a)$ such that the same is true of all $a^{\prime} \models \theta$.

To the best of my knowledge the question of definability of orthogonality in the context of amalgamation constructions has been addressed explicitly only in [HI03] (in a different setting) where it is the main technical tool required for the construction, and in $\S 7$ of [HH06a]. In the original works of Hrushovski's non-orthogonality of simple pre-algebraic $n$-types amounted to the action of $\operatorname{Sym}(n)$ on the variables of $p$, as can be inferred, from the proof of the Algebraic Amalgamation Lemma of [Hru93]. Again, slightly changed in formulation, this lemma says:

Lemma 2.9. Let $A \leq B_{1}, B_{2} \leq M=T_{\infty}, B_{1} / A$ simply pre-algebraic. Let $E=B_{1} \oplus_{A} B_{2}$ be the free amalgam and assume that $p \in S(E)$ is pre-algebraic with $\mu>\delta(A)$ pairwise disjoint realization in $E$ then either $p=\operatorname{tp}\left(B_{1} / A\right)$ for some enumeration of $B_{1} \backslash A$ or $\operatorname{dim}_{B_{2}} p=\operatorname{dim}_{E} p$.

In this and in similar cases definability of orthogonality trivializes. In the case of the fusion over a vector space $V_{F}$, in some sense, $V$-linear dependence replaces some of the roles of equality in fusions over $\{=\}$. It is therefore natural that in this setting a similar claim is true, but with the action of $\mathrm{AGL}_{n}(F)$ (the group of invertible affine transformations of $V_{F}^{n}$ ) on $p$. This is proved, essentially, in Lemma 6.4 of [BMPZ05b]. The uniform definability of orthogonality follows easily. Reformulating (and slightly weakening), it says:

Lemma 2.10. Let $A, B_{1}, B_{2}, E$ be as above. Let $p \in S\left(B_{2}\right)$ be a simply prealgebraic modular type. There exists a natural number $\lambda(p)$ such that if there is a $\lambda(p)$-Morley-sequence in $p$ contained in $E$ then either $\operatorname{dim} B_{2} p=\operatorname{dim}_{E} p$ or there is $H \in \mathrm{GL}_{n}(F)$ and $a \in B_{2}$ such that $\operatorname{tp}\left(\left(H\left(B_{1}\right)+a\right) / A\right)=p$ (for some enumeration of $B_{1} \backslash A$ ).

Note that in both cases the above lemmas give us more information than the definability of orthogonality. They give us the number $\lambda(p)$ which is a bound on the length of Morley sequences in $E$ of any strongly minimal $p \in S(E)$ which is not based on $B_{2}$ (or $B_{1}$ ). These bounds will be most significant in the next subsection.

Having obtained the definability of orthogonality, we now have a better chance of getting definable control over non-orthogonality classes. Following 
(2) of the guidelines described in Subsection 2.3 it is usually useful to definably separate affine types from modular types. In most worked out examples (including - somewhat surprisingly, maybe - [BHMPW06]) all strongly minimal types are trivial, whence modular (in this latter example, this is a key feature in making the collapse possible). In the case of the fusion over a vector space (as in the case of the Red fields of [BMPZ05a]) this is not the case, and some work is needed. This appears as Lemma 7.8 of [HH06a]. In [BMPZ05b] this corresponds to the distinction made between the so called coset types and other types, which is taken care of in $\mathrm{C}(\mathrm{v})$ of the definition of codes.

For technical reasons it is more convenient to be able to definably collect simply pre-algebraic types. This means that for every simply pre-algebraic type $p \in S(B)$ we want a formula $\varphi(x, b) \in p$ and $\psi \in \operatorname{tp}(b / \emptyset)$ such that for every $b^{\prime}=\psi$ the formula $\varphi\left(x, b^{\prime}\right)$ is stationary with a simply pre-algebraic generic type. This is easily seen to be equivalent to definability of strong minimality in $T_{\infty}$. In some cases, this may not be needed at all (see [HH06b]) and in general it will suffice to have a rank preserving expansion of $T_{\infty}$ with this property. It is not known if in the absence of such an expansion the construction cannot be carried out. It is also worth mentioning that for fusion constructions this technical point is the only place DMP of the fused theories is need - it gives definability of strong minimality in $T_{\infty}$. It should not be hard to check that the converse is also true. In the fusion over a vector space, definability of strong minimality in $T_{\infty}$ is taken care of by $\mathrm{C}(\mathrm{i})-\mathrm{C}$ (iii) of the definition of codes (and the subsequent proof that codes exist). The proof of this part in the definition of codes amounts to not much more than definability of Morley rank and degree in each of the fused theories and is very close in spirit to the first order axiomatization of $T_{\infty}$ as discussed in Subsection 2.1.

Finally, we have to decide how to choose representatives of the non orthogonality classes (of strongly minimal types). If we enumerate all $\varphi(x, y)$ such that whenever $\varphi(x, b)$ is non-empty it isolates a simply pre-algebraic type, then using induction and uniform definability of orthogonality, it is not hard to make sure that if $\varphi_{i}(x, b) \perp \varphi_{j}(x, d)$ for some $b$ and all $j<i, d$ we can find a restriction $\tilde{\varphi}_{i}(x, y)$ of $\varphi_{i}(x, y)$ such that $\tilde{\varphi}_{i}(x, b) \sim \varphi_{i}(x, b)$ and for all $b^{\prime}$ for which $\tilde{\varphi}_{i}\left(x, b^{\prime}\right)$ is non-empty the same is true (i.e. $\left.\tilde{\varphi}_{i}\left(x, b^{\prime}\right) \perp \varphi_{j}(x, d)\right)$. It is harder to control non-orthogonality within different instances of $\varphi(x, y)$ itself. As a rule, assuring that exactly one representative is chosen for each non-orthogonality class is non-trivial and has to be done in $T^{e q}$, as this is done in $\S 2.5$ of [CH03] (and see also Lemma A.2 of [HH06a] for the case of the fusion over sub-languages). A different approach, which was taken in [BMPZ05b] (following the simpler treatment in [Hru92]), is to make sure that given a strongly minimal type $p:=p_{a}$, a formula $\varphi(x, a) \in p$ is chosen in such a way that:

(1) For all "unavoidable" $p^{\prime} \not \perp p$ there is some $a^{\prime}$ such that $\varphi\left(x, a^{\prime}\right) \in p^{\prime}$. 
(2) For all other $p^{\prime} \not \perp p$ there is no such $a^{\prime}$.

In [BMPZ05b] this is achieved in $\mathrm{C}(\mathrm{vi})$ and $\mathrm{C}$ (vii) of the definition of codes. Luckily, the "unavoidable" types can only appear in finitely many guises, allowing for a definable control over them. Specifically, if $\varphi_{c}(x, y)$ is a code then:

$\mathbf{C}(\mathbf{v i})$ : For all $b$ and $m$ the set defined by $\varphi_{c}(x+m, b)$ is encoded by $\varphi_{c}$.

C(vii): There is a subgroup $G_{c}$ of $\mathrm{GL}_{n}(F)$ such that:

(1) For all $H \in G_{c}$ and all nonempty $\varphi_{c}(x, b)$ there is a unique $b^{H}$ such that $\varphi_{c}(H x, b) \equiv \varphi\left(x, b^{H}\right)$.

(2) If $H \in \mathrm{GL}_{n}(F) \backslash G_{c}$ then no nonempty $\varphi_{c}(H x, b)$ is encoded by $\phi_{c}$.

and the fact that this can be achieved is not a difficult consequence of compactness.

Since we are following [BMPZ05b] as a main example, in the present discussion we will adopt the approach which does not lead us into the imaginary world.

Gathering all this together we obtain a collection $\mathcal{C}$ of families of strongly minimal sets $\left\{\varphi_{i}(x, y)\right\}_{i<\omega}$ such that for every simply pre-algebraic type $p$ there exists a unique $i<\omega$ and some $b$ such that $\varphi_{i}(x, b) \not \perp p$ and the set $\left\{b^{\prime}: \varphi_{i}\left(x, b^{\prime}\right) \not \perp p\right\}$ is uniformly definable. This gives us an analogue of a representative set of all standard systems of geometries (Definition 2.5.6 of [CH03]), which play a central role in the construction of envelopes in Lie coordinatizable structures. The significant difference (which causes many technical problems) from the $\aleph_{0}$-categorical case is that the domain of our standard systems of geometries (in this context usually better known as codes) is not a complete type.

We note that the complexity of the construction of a standard system of geometries (codes) depends mostly on the possible complexity of nonorthogonality and not (directly) on the geometry of the strongly minimal sets. Thus the codes appearing in [Hru92], e.g. are relatively simple, whereas those appearing in [BHMPW06] are as complicated as those appearing in fusion over a vector space, despite of the fact than in both cases all strongly minimal types are trivial.

2.6. Pseudo-envelopes. From now on we fix a set $\mathcal{C}$ of representatives of all standard system of geometries satisfying all the properties discussed in the previous subsection. We remind the following from [CH03]:

Definition 2.11. (1) An approximation to a geometry of a given type is a finite or countable dimensional geometry of the same type.

(2) A dimension function is a function defined on $\mathcal{C}$, with values isomorphism types of approximations to canonical projective geometries of the given type. (This is actually determined by a dimension, and the type.) 
(3) If $\mu$ is a dimension function, then a $\mu$-envelope is a subset $E$ satisfying the following three conditions:

(a) $E$ is algebraically closed in $M$ (not $M^{e q}$ );

(b) For $c \in M \backslash E$, there is a standard system of geometries $J$ with domain $A$ and an element $b \in A \cap E$ for which $\operatorname{acl}(E c) \cap J_{b}$ properly contains acl $(E) \cap J_{b}$;

(c) For $J$ a standard system of geometries defined in $A$ and $b \in$ $A \cap E, J_{b} \cap E$ has the isomorphism type given by $\mu(J)$.

The two main properties of envelopes which are of interest to us are their existence and homogeneity for every dimension function $\mu$. Our aim is to suggest a definition of envelopes suitable for the present context, such that for some finite $\mu$ (i.e. such that $\mu(J)$ is finite for all $J \in \mathcal{C}$ ) the same will be true.

A major concern in the present context is that we want envelopes to be uncountably categorical (in the original setting the aim was to show envelopes were finite, provided $\mu$ was) so we have to take care that the good properties of our envelopes will be preserved under taking elementary extensions and substructures. We will tackle this last problem by assuring that our envelopes have a reasonable first order axiomatization.

As we have already hinted above, a major problem in carrying out the plan of constructing envelopes is that the domain of our system of standard geometries are not complete types. This is a serious problem for the following reason. If $J \in \mathcal{C}$ and $a \in \operatorname{dom} J$ then $J_{a}$ is a totally categorical geometry. Hence, there is a finite set of formulae $\varphi_{1}\left(x, a_{1}\right), \ldots, \varphi_{k_{a}}\left(x, a_{k}\right)$ generating the structure on $J_{a}$. As long as the $\varphi_{i}$ can be chosen uniformly in $a$, there will be no problem specifying in a first order way that for all $a^{\prime} \in \operatorname{dom}(J) \cap A$ we have $\operatorname{dim}_{J_{a^{\prime}}} N=\mu(J)$ (once we specify $\operatorname{dom}(J)$ we would only have to say that there is a Morley sequence of length $\operatorname{dim} \mu(J)$ in $J_{a}(N)$ whose algebraic closure is the whole of $\left.J_{a}(N)\right)$. But if the structure of $J_{a}$ is not uniform in $a$, this will not be a first order statement ${ }^{8}$. For future reference it will be useful to introduce:

Definition 2.12. A system of standard geometries $\mathcal{C}$ admits a uniform structure, if for any $J \in \mathcal{C}$ there are $\varphi_{1}(x, y), \ldots, \varphi_{n_{J}}(x, y)$ such that for all $a \in$ dom $J$ the structure of $J_{a}$ is generated by $\varphi_{1}(x, a), \ldots, \varphi_{n_{J}}(x, a)$.

Our first goal is therefore to define pseudo- $\mu$-envelopes in $T_{\infty}$ where the type of the geometry approximating $J_{a}$ will not be specified, but only a good enough definable approximation thereof. Fortunately, [CH03] provides a characterization of envelopes which is easier to handle in our context:

Fact 2.13. Let $M$ be a Lie coordinatizable structure. If $E=\operatorname{acl}(E) \subseteq M$ is maximal such that for every $J \in \mathcal{C}$ and $a \in \operatorname{dom}(J)$ the geometry $J_{a}(E)$ embeds into $\mu(J)$ then $E$ is a $\mu$-envelope.

\footnotetext{
${ }^{8}$ This is equivalent to the non-definability of strict minimality in $T_{\infty}^{e q}$
} 
It is now natural do define:

Definition 2.14. Let $\mu: \mathcal{C} \rightarrow \mathbb{N} \cup\{\infty\}$. An algebraically closed structure $E$ is a pseudo $\mu$-envelope if it is maximal such that for every $J \in \mathcal{C}$ and every $a \in \operatorname{dom}(J) \cap E$ there is no pseudo $\mu(J)$-Morley sequence of length $\mu(J)$ in $J_{a}(E)$.

It remains, of course, to define pseudo $n$-Morley sequences, which is a rather tedious and technical job. We will not give the whole set of requirements (which may vary from structure to structure) but review only the most important ones:

Fix a stationary type $p$ and denote $p^{\otimes n}$ the type of an independent set of $n$ realizations of $p$. A pseudo $n$-Morley sequence in $p$ is a formula $\psi_{n} \in p^{\otimes n}$ such that for $\left(a_{1}, \ldots, a_{n}\right) \models \psi_{n}$ :

(1) $C b(p) \subseteq \operatorname{dcl}\left(a_{i_{1}}, \ldots, a_{i_{k}}\right)$ for some $k$ depending only on $p$ (but not n) and for all $1 \leq i_{1}<\cdots<i_{k} \leq n$. In particular, $n$ has to be large enough.

(2) $\left(a_{\sigma(1)}, \ldots, a_{\sigma(n)}\right) \models \psi_{n}$ for any $\sigma \in \operatorname{Sym}(n)$.

(3) if $\left.b \models p\right|_{\left(a_{1}, \ldots, a_{n}\right)}$ then $\left(a_{1}, \ldots, a_{i-1}, b, a_{i+1}, \ldots, a_{n}\right) \models \psi_{n}$ for all $1 \leq$ $i \leq n$. Also $\left(a_{1}, \ldots, a_{n}, b\right) \models \psi_{n+1}$.

(4) if $n-1$ is large enough then $\left(a_{1}, \ldots, a_{n-1}\right)=\psi_{n-1}$.

(5) There are no obvious dependencies in $a_{1}, \ldots, a_{n}$ over $C b(p)$ (e.g. they are pairwise disjoint and in the case of the fusion over a vector spaces they are linearly independent with respect to that vector space).

(6) In most cases it will be more convenient to choose $\psi_{n}$ quantifier free in the natural language of $T_{\infty}$.

In [Hru93] pseudo Morley sequences (in simply pre-algebraic types) are just (long enough) sequences of pairwise disjoint realizations of the type. In [Hru92] the definition is slightly more complicated and is practically equivalent to (1)-(5), which are given as part of the definition of 2-codes. In [BMPZ05b] so called difference sequences, as defined in $\S 3$ are pseudo-Morley sequences (if we restrict our attention - as we may - to modular types) with quite a few additional properties which are irrelevant at the moment, and will be referred to below. Since the definition of difference sequences involves many technical details, we will not give it here.

2.7. Overtaking obstructions. Recall that we fixed a system $\mathcal{C}$ of standard geometries. By definition, for every function $\mu$ (and any definition of pseudo $\mu$-Morley sequence) pseudo $\mu$-envelopes (with respect to $\mathcal{C}$ ) exist. Our problem is to show that they are homogeneous (and elementary).

We will do it by giving an explicit axiomatization of the theory of pseudo $\mu$-envelopes (for appropriately chosen $\mu$ ). The first approximation to an axiomatization of pseudo $\mu$-envelopes would be:

(1) $T_{\infty}^{\forall}$

(2) For every model $E$, every $J \in \mathcal{C}$ and $a \in \operatorname{dom}(J) \cap E$ there is no pseudo $(\mu(J)+1)$-Morley sequence in $J_{a}(E)$. 
(3) Every model is (self-sufficient and) algebraically closed in the sense of $T_{\infty}$.

(4) For every model $E$, every $J \in \mathcal{C}$ and every $a \in \operatorname{dom}(J) \cap E$ there exists a pseudo $\mu(J)$-Morley sequence in $J_{a}(E)$.

(5) For every model $E$, every affine geometry definable over $E$ has a point in $E$.

Remark 2.15. In general, it is more natural to define the envelope of a set $X$, in which case something has to be said concerning the realizations of $p_{\omega}$ in the envelope of $X$. In the present context, the most natural (conforming also with the standard definition) would be to require that $E(X) \downarrow_{X} a$ for all $\left.a \models p_{\omega}\right|_{X}$. As we have already said before - since $p_{\omega}$ can take care of itself - we will ignore this part in our treatment of envelopes.

Like the situation in [CH03], these properties (striking out "pseudo" whenever it appears) characterize $\mu$-envelopes, in the present context as well. However, as stated, they will not, in general, be consistent. To better understand the problem consider $E_{0}$ satisfying (1)-(3). Let $J \in \mathcal{C}$ and $a \in \operatorname{dom}(J) \cap E_{0}$ be such that the longest pseudo Morley sequence in $J_{a}\left(E_{0}\right)$ has length $\nu\left(J_{a}\right)<\mu(J)$. Let $b=\left.J_{a}\right|_{E_{0}}$ and $E:=\operatorname{acl}\left(E_{0} b\right)$. Does E satisfy (3)? By the construction of our system of standard geometries, for all $J^{\prime} \in C$ and all $a^{\prime} \in \operatorname{dom}(J) \cap E_{0}$ we have

$$
\operatorname{dim}_{E} J_{a}^{\prime} \leq \operatorname{dim}_{E} J_{a}^{\prime}+1
$$

and strict inequality holds unless $J=J^{\prime}$ and $J_{a} \not \perp \quad J_{a^{\prime}}$. Since $E_{0}=$ $\operatorname{acl}_{T_{\infty}}\left(E_{0}\right)$ it follows from the above that $J_{a}^{\prime}\left(E_{0}\right)=J_{a_{0}}^{\prime}\left(E_{0}\right)$ for all other $J^{\prime}$ and $a^{\prime} \in \operatorname{dom}(J) \cap E_{0}$ implying that (3) holds of $J_{a^{\prime}}^{\prime}$. In fact, since our standard geometries are all modular, if $J=J^{\prime}$ and $J_{a} \not \perp J_{a^{\prime}}$ we must have equality in $(*)$. It is usually not hard to check in that case - with a reasonable definition of pseudo Morley sequences - that for every such $a^{\prime}$ we have in $E$ sequences of length $\nu\left(J_{a}\right)+1 \leq \mu(J)$. However, it is not at all clear (and in fact, not in general true, see the example of $\S 4$ of $[\mathrm{BH}]$ ) that the same holds of any $a \in \operatorname{dom}(J) \cap E$ (but not necessarily $a \in E_{0}$ ).

Since (1)-(3) are obviously not strong enough (as they say nothing on the maximality of pseudo-envelopes) and (1)-(4) are inconsistent we have to correct our axioms into something of the flavor of:

$\left(^{\prime}\right)$ : For every model $E$, every $J \in \mathcal{C}, a \in \operatorname{dom}(J) \cap E$ and $k<\mu(j)$, if $b_{1}, \ldots, b_{k}$ is a pseudo $k$-Morley sequence in $J_{a}(E)$ either:

(a): there exists $b_{k+1} \in J_{a}(E)$ such that $\left(b_{1}, \ldots, b_{k}, b_{k+1}\right) \models \psi_{k+1}^{J}$; or

(b): for every generic $b \in J_{a}$ there exists $J^{\prime} \in \mathcal{C}$ and $a^{\prime} \in \operatorname{dom} J^{\prime}$ such that in $\operatorname{acl}_{T_{\infty}}(E b)$ there exists a pseudo $\left(\mu\left(J^{\prime}\right)+1\right)$-Morley sequence in $J_{a^{\prime}}^{\prime}$.

Our problem now is to show that (4')-b can be stated in a first order way. Towards this end we will introduce the only new definition of this text (see Appendix A of [HH06a] for more details). 
Definition 2.16. $\quad(1)$ Let $a \in \operatorname{dom}(J)$ for some $J \in \mathcal{C}$. Say that $J_{a}$ admits an obstruction if there exists some $B=\operatorname{acl}(B)$ such that for every generic $b \in J_{a}$ there exists some $J^{\prime} \in \mathcal{C}$ and $d \in\left(\operatorname{dom}\left(J^{\prime}\right) \cap\right.$ $\operatorname{acl}(B b)) \backslash\left(\operatorname{dom}\left(J^{\prime}\right) \cap B\right)$ such that $\left(J_{d}^{\prime} \perp J\right.$ and $) \operatorname{dim}_{\mathrm{acl}(B b)} J_{d}^{\prime}>0$. Call $\operatorname{dim}_{\mathrm{acl}(B a)} J_{d}^{\prime}$ the size of the obstruction.

(2) $J_{a}$ admits bounded obstruction if there is a bound on the size of the obstructions it admits.

(3) $J$ admits bounded obstructions if $J_{a}$ does for all $a \in \operatorname{dom} J$ and the bound is uniform (in a).

It follows almost immediately from the definition of Lie coordinatizable structures, that any family of standard geometries does not admit any obstructions at all (i.e. all obstructions are uniformly bounded by 0 ). The same proof works in the present context. As explained in Subsection 2.5, the problem with translating the proof to the case of pseudo-envelopes is that if $c \in \operatorname{dom} J$, it may well be that $\operatorname{acl}(c)$ contains fairly long (unboundedly as $c$ varies over $\operatorname{dom} J$ ) pseudo Morley sequences (so it will not be appropriate to call these, "pseudo-obstructions", since they actually cause much more trouble than obstructions. But we'll do it nonetheless !).

In [Has04] this problem is bypassed, by assuming the existence of a system of standard geometries admitting uniform structure. As we have already said, this assumption is not valid in $T_{\infty}$ for most amalgamation constructions. The solution is to prove enough uniformity of structure in order to uniformly bound the size of pseudo-obstructions. In practice, what we will prove is the existence of a function $c: \mathcal{C} \rightarrow \mathbb{N}$ and for every $J \in \mathcal{C}$ a natural number $B(J)$ such that for every $J^{\prime} \in \mathcal{C}$ the size of a possible pseudo obstruction to $J$ witnessed by an element from $J^{\prime}$ is bounded by $B(J)+c\left(J^{\prime}\right)$. Before we proceed to explain how this can be achieved, we show why this would be enough.

Choose any enumeration of $\mathcal{C}$. Let $E_{0}=(1)-(3), b \in \operatorname{dom} J^{i}$ and $a \in J_{b}^{i}$ generic over $E_{0}$. By our assumption the size of a $J_{i}$ obstruction witnessed by some $J^{\prime}$ is bounded by $B\left(J^{i}\right)+c\left(J^{\prime}\right)$. Choose any function $\mu^{*}: \mathcal{C} \rightarrow \mathbb{N}$ which is finite to one, and set $\mu(j)=\mu^{*}(j)+c\left(J^{j}\right)$. So there are only finitely many $j \in \mathbb{N}$ such that:

$$
B\left(J^{i}\right)+c\left(J^{j}\right) \geq \mu(j)=\mu^{*}(j)+c\left(J^{j}\right) .
$$

So for such a choice of $\mu$, for a given $J \in \mathcal{C}$ there are only finitely many $J^{\prime} \in \mathcal{C}$ which may witness the existence of a pseudo-obstruction, and axiom scheme 4'-(b) readily becomes elementary.

So we now turn back to the proof of the above form of uniform boundedness of pseudo obstructions. The idea is to show that if $E=\operatorname{acl}(E), J \in \mathcal{C}$, $a \in \operatorname{dom}(J) \cap E$ and $b \in J_{a} \backslash E$ then there exists a function $\Delta: \mathcal{C} \rightarrow \mathbb{N}$ such that if $b_{1}, \ldots, b_{\Delta\left(J^{\prime}\right)}$ is a pseudo Morley sequence in $J_{c}^{\prime} \cap \operatorname{acl}(E a)$ for some $J^{\prime} \in \mathcal{C}$ and $c \in \operatorname{dom} J^{\prime}$ then either $J_{c}^{\prime} \not \perp E$ or $J_{c}^{\prime} \not \perp \operatorname{acl}(a b)$. This is enough, since if $J_{c}^{\prime} \not \perp E$ then $c \in E$ (because $E=\operatorname{acl}(E)$ ) and therefore it must be that $J^{\prime}=J$ and $c=b$, so this is not an obstruction. If, on the other hand, 
$J_{c}^{\prime} \not \perp$ acl $(a b)$, we may assume that $c \notin E$ so there is a uniform bound $c\left(J^{\prime}\right)$ on the length of a pseudo Morley sequence in $J^{\prime}$ containing only elements which meet $\operatorname{acl}(b)$ (otherwise this will contradict either the assumption that $c \notin \operatorname{acl}(b) \subseteq E$ or the fact that $\operatorname{acl}(b) \leq M)$. So all but $c\left(J^{\prime}\right)$ of the elements in the sequence witnessing the pseudo-obstruction must lie in $\operatorname{acl}(a b) \backslash \operatorname{acl}(b)$, and it remains to check that the number of such elements is bounded by a function of $|a|$ (which depends, among others, on the definition of pseudoMorley sequences). In the case of fusion over a vector space this bound is simply $|a|$.

So finally, we are reduced to showing that indeed (with the same notation) $J_{c}^{\prime} \not \perp E$ or $J_{c}^{\prime} \not \perp \operatorname{acl}(a b)$. The key observation is that the geometries (but not the pre-geometries!) of strongly minimal types in $T_{\infty}$ are determined by the quantifier free formulae (in the natural language of $T_{\infty}$ ), and that the quantifier free structure of standard geometries is uniform ${ }^{9}$.

To state this more precisely, it will be useful to introduce for a stationary type $p$ the notation $q f p^{\otimes n}:=\left\{\varphi \in p^{\otimes n}: \varphi\right.$ quantifier free $\}$. With this notation, Lemma 5.4 in [BMPZ05b], translated into a more standard model theoretic language, says:

Lemma 2.17. Let $M \leq N$ and $J \in \mathcal{C}$. There exists a function $\lambda(n, J)$ such that for $b \in \operatorname{dom}(J)$ and distinct $a_{1}, \ldots, a_{\lambda(n, J)} \in J_{b}(N)$ either $J_{b} \not \perp M$ or there are $1 \leq i_{1}<\cdots<i_{n} \leq \lambda(n, J)$ such that $\left.\left(a_{i_{1}}, \ldots, a_{i_{n}}\right) \models q f J_{b}^{\otimes n}\right|_{M}$.

This is a simple corollary of the total categoricity of strongly minimal sets in $T_{\infty}$. The same lemma appears, and has an as important role in the construction of the bad field of [BHMPW06]. In the latter case the proof is somewhat harder, mostly due to the fact that local finiteness of the strongly minimal sets in $T_{\infty}$ is far from obvious.

The desired conclusion now follows rather easily, but since it uses quite heavily the specific construction, we will avoid the details. In [BMPZ05b] this appears as Lemma 7.3 and in [BHMPW06] it is Lemma 9.1, with a similar proof.

It should be clear by now that we can use the same results also to obtain the analogous correction of (5):

(5'): Every affine space $(A, V)$ definable over $E$ has a point in $E$, unless for every generic $b \in A$ one of possibly finitely many (definable families) of obstructions occur.

So in order to check that this axiom is consistent with (1)-(4') we simply have to make sure that if no obstruction occur and $(A, V)$ does not have a point in $E$ then $\operatorname{acl}(E b)$ still satisfies (1)-(4') for any choice of $b \in A$ generic

\footnotetext{
${ }^{9}$ This statement is not quite precise, see e.g. [HH06b]. Moreover, it depends heavily on $\mathcal{L}\left(T^{\forall}\right)$, and if $T_{\infty}$ happens to have $\mathrm{QE}$ in that language, will generally not be true. Suffice it if we say that in all known examples there exists $\mathcal{L}^{\prime} \subseteq \mathcal{L}\left(T_{\infty}\right)$ such that the geometry of all strongly minimal sets is determined by their $\mathcal{L}^{\prime}$ structure and in which we have uniformity of structure.
} 
over $E$. This is an almost immediate corollary of the uniqueness of parallel lines (see $\S 3$ of [CH03] for the details).

2.8. Concluding remarks. We have shown in the last subsection that for $\mu$ growing fast enough, pseudo $\mu$-envelopes are first order axiomatizable. Denoting the resulting theory $T_{\mu}$ it is now a straightforward exercise to ascertain that models of $T_{\mu}$ are homogeneous. The proof proceeds by back and forth. The point is that if $E$ is a pseudo envelope, $A_{i}=\operatorname{acl}_{T_{\infty}}\left(A_{i}\right)$ (for $i=1,2)$ are small and $f: A_{1} \rightarrow A_{2}$ is a partial isomorphism, then for every $a \in E$ there exists $\bar{a} \subseteq E$ such that $a \in \bar{a}$ and $\bar{a} / A_{1}$ is prealgebraic. The analysis of the previous subsection shows that the only way there could not be a solution of $f(\operatorname{tp}(\bar{a} / A 1))$ in $E$ is if there is an obstruction witnessed already in $\operatorname{acl}_{T_{\infty}}\left(f\left(C b\left(\bar{a} / A_{1}\right) \cup\{\bar{a}\}\right)\right.$ ) (where $f(a)$ is a any generic realization of $\left.f\left(\operatorname{tp}\left(\bar{a} / A_{1}\right)\right)\right)$, which cannot be, as this would imply an obstruction already existed in $A_{1}$.

Therefore, $T_{\mu}$ eliminates quantifiers (relative to $T_{\infty}$ ) whence it inherits the coordinatization of $T_{\infty}$ and consequently has a unique non-algebraic regular type (all the regular types in $T_{\infty}$ orthogonal to $p_{\omega}$ have dimension bounded by $\mu$ in all models - hence they are algebraic). With the coordinatization $T_{\mu}$ inherits also the $\omega$-stability of $T_{\infty}$, so pseudo envelopes are uncountably categorical, and $T_{\mu}$ is the collapse of $T_{\infty}$.

The most natural question at this stage is of course to understand the geometry of the strongly minimal set (corresponding to the unique nonalgebraic type) obtained in this way. To answer this question, it will be more convenient to work in a saturated $E \models T_{\mu}$, such that $E \leq M=T_{\infty}$ (recall that for the construction of $T_{\mu}$ we worked in an existentially closed model of $T_{\infty}$, so the resulting envelope could not be saturated). Choosing a transcendence base $I \leq E$, we get that $E$ is the pseudo $\mu$-envelope of $I$. It is now not hard to check, that any finitary property of the geometry of $p_{\omega}$ will be preserved in the geometry of $T_{\mu}$, provided that $\mu$ is large enough. More precisely, consider any set $a_{1}, \ldots, a_{k} \in I$ then

$$
\operatorname{cl}_{p_{\omega}}\left(a_{1}, \ldots, a_{k}\right) \cap E=\operatorname{acl}_{T_{\mu}}\left(a_{1}, \ldots, a_{k}\right) \backslash \operatorname{acl}_{T_{\mu}}(\emptyset) .
$$

This readily implies, that at least for generic $\mu$ (in any reasonable definition of the term) the geometrical properties of $p_{\omega}$ are reflected in the unique generic type of $T_{\mu}$. For example, it is clear that if $p_{\omega}$ is $n$-ample for some $n$ (see [Pil00] for the definition) then so is $T_{\mu}$. Note, however, that in general (see, e.g. $\$ 5.2$ of [Hru93]) $\mu$ may be recoverable from the geometry of $T_{\mu}$, so that the geometry of $p_{\omega}$ will not be isomorphic to that of $T_{\mu}$.

So the role of the free construction is to provide us with the theory $T_{\infty}$ where the collapse is taking place and the local geometry (of its unique regular type of infinite rank) is the one ultimately determining the geometry of the collapse. It is a thorough (though more often than not implicit) analysis of the geometric properties of $T_{\infty}$ which tells us how to perform the collapse, which can be viewed as a smooth approximation of $T_{\infty}$. 
It is perhaps not just a coincidence that both the free construction and the collapse are (or at least can be) obtained by means of amalgamation. But this similarity is misleading - the two constructions are genuinely different. I hope that this section of the paper has made it clear that there is a good model theoretic theory underlying the collapse. I hope that the next section will help me convince that the true challenge, the terra incognita, lies in the realm of free constructions.

\section{Some Old OPEn QUestions}

This section is dedicated to the presentation of some, in my view, important open questions related to Hrushovski's construction. Some of the problems may be quite hard, but others seem accessible, possibly even easy. Throughout, all theories will be in countable languages.

3.1. Geometry. The questions in this subsection are motivated mainly by:

Problem 3.1. Is there a classification of strongly minimal geometries.

But also by the challenge of constructing a bad group of finite Morley rank. Already the first step in Problem 3.1 seems rather hard:

Problem 3.2. Formulate a conjecture on the classification of strongly minimal geometries.

A reasonable approach to the problem would be a classification of strongly minimal sets according to the complexity of their geometries. A possible measure of complexity can be found in Pillay's hierarchy of $n$-ample geometries proposed in [Pil00]. Pillay's conjectural hierarchy has two major drawbacks:

- It does not offer any gauge of complexity for structures interpreting infinite fields (as such structures are $n$-ample for all $n$ ).

- All known strongly minimal structures either interpret an infinite field or fall into one of the three first classes in the infinite hierarchy.

A partial remedy to the first of these problems could be to classify the strongly minimal geometries in terms of (countable) collections of "basic" types of geometries (say the $n$-ample non $(n+1)$-ample geometries together with the geometries of algebraically closed fields). But this does not settle the question of the sort of classification such collections of geometries may give us.

Recovering the geometry of a strongly minimal set from a set of reducts of lower complexity may be an over ambitious goal, as the following example shows. Consider $T$, a strongly minimal fusion (over $\{=\}$, say) of two algebraically closed fields, $T_{1}, T_{2}$. The natural choice of a set of geometries associated with $T$ is given by the geometries of $T_{1}, T_{2}$. However, recovering the geometry of $T$ from the data might call for additional information concerning the $\mu$-function used in the collapse (see, e.g. $\S 5$ of [Hru93]). 
In the above example the more natural geometry to associate with $T_{\mu}=$ $T_{1} \oplus_{\mu} T_{2}$ is the local geometry of $p_{\omega}$ in the corresponding free fusion $T_{\infty}:=$ $T_{1} \oplus_{\infty} T_{2}$. In addition, it is easy to verify that if $T_{\mu}=T_{1} \oplus_{\mu_{1}} T_{2} \oplus_{\mu_{2}} T_{3}$ the local geometry of $T_{\infty}:=T_{1} \oplus_{\infty} T_{2} \oplus_{\infty} T_{3}$ is fully determined by $T_{1}, T_{2}, T_{3}$ (and in particular does not depend on the order in which the theories are fused). So we define:

Definition 3.3. Let $\left\{T_{i}\right\}_{i \in I}$ be a countable collection of strongly minimal sets in countable (disjoint) languages, $\left\{\mathcal{G}_{i}\right\}_{i \in I}$ their associated geometries. The free amalgam $\mathcal{G}:=\bigoplus_{i \in I} \mathcal{G}_{i}$ is the local geometry of $T_{\infty}:=\bigoplus_{i \in I} T_{i}$.

A Combinatorial geometry $\mathcal{G}$ is a free amalgam, if it is obtained as the free amalgam of n-ample (possibly unbounded $n$ ) strongly minimal geometries and the geometries of algebraically closed fields.

To clarify the above definition we need a couple of observations:

Remark 3.4. (1) Recall that if $T_{1}, T_{2}$ are strongly minimal geometries then $T_{2} \oplus_{\infty} T_{2}$ always exists, regardless of DMP, which is only needed for the collapse.

(2) The pre-dimension function for the free fusion of countably many theories at one go is given by $\delta(A):=|A|+\sum_{i \in I}\left(\operatorname{MR}_{i}(A)-|A|\right)$. By requiring that $\delta(A) \geq 0$ (which is an elementary property) we assure that the support of this last sum is finite.

(3) The standard proof of $\omega$-stability of the resulting theory $\bigoplus_{i \in I} T_{i}$ goes through unaltered.

Thus, we have a canonical way of associating with a given collection of strongly minimal geometries (possibly with repetitions ${ }^{10}$ ) a combinatorial geometry (which need not, in general, be that of a strongly minimal set). It may therefore be reasonable to try and classify the geometries of strongly minimal sets using those of free amalgams of strongly minimal geometries.

This raises naturally, the question already hinted to in the end of the previous section, concerning the relation between the local geometry of $T_{\infty}$ and the geometry of $T_{\mu}$. As already mentioned, the geometry of $T_{\mu}$ can be viewed as obtained from the one in $T_{\infty}$ by reducing to an appropriate subset of realizations of $p_{\omega}$. Although this is not quite a satisfactory characterization, the following can be asked:

Problem 3.5. Is every strongly minimal geometry an infinite homogeneous subset (with the induced closure operator) of a free amalgam?

It may well be that the definition of freely amalgamated geometries will have to be modified to encompass even the known constructions. The obvious test cases are:

Problem 3.6. (1) Let $T:=T_{1} \oplus_{T_{0}}^{\infty} T_{2}$ be the (free) fusion of the strongly minimal theories $T_{1}, T_{2}$ over their common totally categorical vector

${ }^{10}$ What is the geometry of the fusion of an algebraically closed field with itself ? 
space $T_{0}$. If $T_{1}, T_{2}$ satisfy the conclusion of the previous problem, does $T ?^{11}$.

(2) Let $T$ be a theory of finite Morley rank with DMP. Let $D(T)$ be a strongly minimal theory interpreting it, as obtained in [Has07]. If every strongly minimal set definable in $T$ satisfies the conclusion of the previous problem, does $D(T)$ ?

(3) As a concrete example of (2) above, let $T$ be the theory consisting of $E$, an equivalence relation with infinitely many classes, each of which supporting (uniformly) a stricture of an algebraically closed field. Let $X$ be the imaginary sort corresponding to $E$ and $T^{\prime}$ the theory $T$ fused with a function $F: E \rightarrow X$ which is a bijection on the E-classes. So $T^{\prime}$ is a strongly minimal theory. Does its geometry come from an amalgamated one?

(4) What about the geometries associated with the (almost strongly minimal) colored fields?

A positive answer (to my mind, not very likely) to the following question, would suggest a more natural formulation of Problem 3.5:

Problem 3.7. Let $T_{\mu}:=T_{1} \oplus_{\mu} T_{2}$. Is the geometry of $T_{\mu}$ locally isomorphic to that of $T_{\infty}$ (i.e. are the two geometries isomorphic after localizing at a set $S$ with $\left.\operatorname{dim}(S)=\operatorname{codim}(S)=\aleph_{0}\right)$.

More generally, we can ask:

Problem 3.8. Is there a canonical way of associating with any strongly minimal geometry an amalgamated geometry, in such a way that the geometry associated to "basic" geometries is basic.

Problem 3.7 generalizes a question form [Hru93]. In $\S 5$ of the paper continuum many non-isomorphic strongly minimal flat geometries are constructed, and the question arises as to whether these geometries are non locally isomorphic. It is also asked whether a continuum of non locally-isomorphic flat strongly minimal geometries exists. To the best of my knowledge these questions are still open.

A related problem, generalizing 3.7, which may be of interest (although it does not seem to have immediate implications on the classification problem) is:

Problem 3.9. Let $\left\{G_{i}\right\}_{i \in I}$ and $\left\{F_{j}\right\}_{j \in J}$ be countable collections of strongly minimal geometries, $\mathcal{G}, \mathcal{F}$ their respective free amalgams. Suppose there is no bijection $f: I \rightarrow J$ such that $\mathcal{G}_{i} \cong \mathcal{F}_{f(i)}$ for all $i \in I$. Can it be that $\mathcal{G} \cong F$. Can they be locally isomorphic if the basic geometries are not?

Note that in the above problem $\mathcal{F}, \mathcal{G}$ need not be amalgamated geometries, as it is not assumed that $\left\{\mathcal{G}_{i}\right\}_{i \in I}$ and $\left\{F_{j}\right\}_{j \in J}$ are "basic" geometries.

\footnotetext{
${ }^{11}$ Recall that under the same assumptions, if in addition $T_{1}, T_{2}$ are one based, then $T_{1} \oplus_{T_{0}}^{\mu} T_{2}$ is 1-based [HH06a] whereas $T_{1} \oplus_{\mu} T_{2}$ - the fusion over $\{=\}$ isn't
} 
Returning to the question of the number of non locally isomorphic strongly minimal geometries, I don't know of a any published proof of the existence of continuum many such. We give here a short proof, based on an idea of Hrushovski's. Recall, from the introduction of [Hru92]: "The geometry [of the fusion] can be viewed as 'relatively flat' over the geometries of the strongly minimal sets, however. In particular, it can be shown that if $G$ is a connected group definable in the strongly minimal amalgam of $D_{1}, D_{2}$ then there exist connected groups $G_{i}$ definable over $D_{i}$ and definable surjective group homomorphism $f: G \rightarrow G_{1} \times G_{2}$ with finite (central) kernel. It would be good to have the sharper result with the arrow reversed $f: G_{1} \times G_{2} \rightarrow G$."

Already from the easier of the above assertions it would follow that if $F_{1}, F_{2}$ are strongly minimal fields of distinct positive characteristics $p_{1}, p_{2}$ (respectively) then any strongly minimal group of characteristic $p_{i}$ interpretable in their fusion $F_{1} \oplus_{\mu} F_{2}$ is a finite cover of $\left(F_{i},+\right)$. For every prime $p$ denote $K_{p}:=\operatorname{acl}\left(\mathbb{F}_{p}\right)$. By induction it would follow that for any (possibly infinite) set $S$ of primes the free fusion $K_{S}:=\bigoplus_{p \in S} K_{p}$ interprets strongly minimal groups of characteristic $p$ precisely for the primes $p \in S$. By Theorem C of [EH95], this gives a continuum of non locally isomorphic strongly minimal geometries, as $S$ varies over subsets of the primes. So we prove:

Claim 3.10. If $G$ is a connected group definable in the strongly minimal fusion $T: D_{1} \oplus_{\mu} D_{2}$ then there exist connected groups $G_{i}$ definable over $D_{i}$ and definable surjective group homomorphism $f: G \rightarrow G_{1} \times G_{2}$ with finite kernel.

idea of the proof. The proof is not hard, so we only give a sketch. The idea is to pull back the group configuration of $G$ to group configurations in $D_{1}$ and $D_{2}$. So let $Q:=\{a, b, c, x, y, z\} \subseteq G$ be a set of $G$-generics forming a group configuration (e.g. $y=a^{-1} x, c=a b$ and $z=b^{-1} a^{-1} x$ ). For simplicity we may assume that $Q \leq M=D_{1} \oplus_{\mu} D_{2}=: T$. By assumption, $y \in \operatorname{acl}_{T}(a x) \backslash\left(\operatorname{acl}_{T}(x) \cup \operatorname{acl}_{T}(a)\right)$, whence $d(y / a x)=0$ and if $\hat{y}_{i}$ are $L_{i^{-}}$ transcendence bases for $y /(a, x)$, we get $\left|\hat{y}_{1}\right|+\left|\hat{y}_{2}\right|=n$ (for $n=\operatorname{length}(y)$, otherwise we would get a contradiction to the assumption that $a, x \in G$ are independent generics).

We can also find $\hat{a}_{i} \subseteq a$ such that $\operatorname{MR}_{i}\left(\hat{a}_{i}\right)=\operatorname{MR}_{i}(a / x, y)=\operatorname{MR}_{i}(y / a, x)$. Similarly, we can find $\hat{x}_{i} \subseteq x$ with the same property. Using the fact that $\operatorname{tp}_{T}(x / y)=\operatorname{tp}_{T}(z / y)$ we find $\sigma \in \operatorname{Aut}(M / y)$ (some saturated $M \models T$ ) such that $\sigma(z)=x$, so we get that $\sigma(b)$ is interalgebraic with $a$ over $x, y$. Pulling back $\hat{a}_{i}$ and $\hat{x}_{i}$ we can find $\hat{b}_{i}$ and $\hat{z}_{i}$ such that the triple $(b, z, y)$ has the same properties with respect to $\left(\hat{b}_{i}, \hat{z}_{i}, \hat{y}_{i}\right)$ as $(a, x, y)$ had with respect to $\left(\hat{a}_{i}, \hat{x}_{i}, \hat{y}_{i}\right)$. By similar arguments we can find a corresponding $\hat{c}_{i} \subseteq c$ with the same properties (with respect to $(a, b, c)$ and $(c, z, x)$ ).

Thus, setting $\hat{Q}_{i}:=\left\{\hat{a}_{i}, \hat{b}_{i}, \hat{c}_{i}, \hat{x}_{i}, \hat{y}_{i} \hat{z}_{i}\right\}$ we get that either $Q \in \operatorname{acl}_{i}\left(\hat{Q}_{i}\right)$, or $Q / \hat{Q}_{i}$ is a group configuration in $L_{i}$. Because $\left|\hat{y}_{1}\right|+\left|\hat{y}_{2}\right|=n$ whereas $\operatorname{MR}_{1}(y)+\operatorname{MR}_{2}(y)>n$ it cannot be that both $Q \in \operatorname{acl}_{1}\left(\hat{Q}_{1}\right)$ and $Q \in$ 
$\operatorname{acl}_{2}\left(\hat{Q}_{2}\right)$, whence at least one actually gives a group configuration. Let $G_{1}, G_{2}$ the corresponding groups (if $Q \in \operatorname{acl}_{i}\left(\hat{Q}_{i}\right)$ we set $G_{i}=\{e\}$, the trivial group).

The claim now follows easily (using the fact that $\hat{Q}_{i}$ is a set of $D_{i^{-}}$ independent generic elements).

Remark 3.11. By similar arguments it can be shown that the only strongly minimal fields interpretable in $T=D_{1} \oplus_{\infty} D_{2}$ are the ones already interpretable in either $D_{1}$ or $D_{2}$ (use the field configuration and the fact that we know it corresponds to the action of a 2-dimensional group on a 1dimensional homogeneous space).

Obtaining the sharper characterization of groups definable in $D_{1} \oplus_{\mu} D_{2}$ as suggested in [Hru92] would, of course, call for a closer analysis of the situation.

It may be worth pointing out, though, that a similar argument would show that if $M$ is a structure of finite Morley rank (with DMP) and $D$ a strongly minimal set interpreting $M$, as obtained by the construction of [Has07], then any group interpretable in $D$ is a finite cover of one already definable in $M$. It seems that the same goes for Ziegler's fusion of structures of finite Morley rank [Zie06], but here the situation is somewhat more delicate. The problem is, that in order to carry the argument through, some care should be taken in handling imaginaries.

Moreover, if $T_{1}:=D_{1} \oplus D_{2}$ is the free fusion of $D_{1}, D_{2}$ (i.e $\mu=\infty$ ) the same should be true. Indeed, in that case, if $G$ is a (connected) group definable in $T$ and $Q$ is a self-sufficient quadrangle as above, then $a \subseteq$ $\operatorname{acl}_{T}(x y) \Longleftrightarrow a \subseteq\langle x y\rangle$ where $\langle\cdot\rangle$ is the transitive closure of closing under $\mathrm{acl}_{1}$ and $\mathrm{acl}_{2}$. Hence this is a special case of the one treated in the proof of Claim 3.10. Interestingly, enough, though, despite of the fact that the claim remains true for the (strongly minimal) fusion over a vector space, it will no longer be true of the free fusion over a vector space. Using [HH06a] it should be a fairly easy exercise to classify the groups of finite rank in this last case.

Finally, note that if $p_{\omega}$ is the (regular) generic type of $T_{\infty}$, any group configuration in $p_{\omega}^{\otimes n}$ would produce a group configuration in $T_{\mu}$ for large enough $\mu$, and therefore must originate in group configurations in $D_{1}, D_{2}$, as in the previous claim.

All this (together with the fact that connected CM-trivial groups of finite Morley rank are nilpotent, [Pi195]) support, the general feeling expressed in the introduction to [BP00]: "...Hrushovski's amalgamation techniques, as they stand at present, produce theories which are CM-trivial or at least CM-trivial over the data (the latter notion has not been made precise yet). So [Pi195] suggests that these methods will not produce new simple groups of finite Morley rank".

Today, this statement is as well accept and us unclear as it was when written in 1998. The main problem with making it more precise is that 
technically it is probably wrong. Let $M$ be a countable (saturated) structure of finite Morley rank. Assume without loss that $T:=\operatorname{Th}(M)$ eliminates quantifiers and is given in a relational language. Let $\mathcal{C}$ be the class of all finite models of $T^{\forall}$. For $A \in \mathcal{C}$ define $\delta(A)=\operatorname{MR}(\operatorname{tp}(f(A)))$ for some elementary embedding $f: A \rightarrow M$. By quantifier elimination $\delta(A)$ is well defined. Note also that quantifier elimination assures that if $A \subseteq B \in \mathcal{C}$ then $\delta(A) \leq \delta(B)$ so $A \subseteq B \Longleftrightarrow A$ is self-sufficient in $B$. Thus $(\mathcal{C}, \subseteq)$ has the amalgamation property, and its Fraïssé limit is $M$.

Therefore, if we translate "Hrushovski's amalgamation techniques" into "Amalgamation constructions in a universal (or inductive) class with predimension", we get that any structure of finite Morley rank can be reconstructed in such a way. Possibly more precise (and certainly more cumbersome) would be the following:

Definition 3.12. A stable pre-dimensioned class is a universal theory $T$ in a language $L$ and a function $\delta: \mathcal{C} \rightarrow \mathbb{N}$, where $\mathcal{C}$ is the set of all finitely generated models of $T$, satisfying:

(1) $\delta$ is L-isomorphism invariant.

(2) $\delta(\emptyset)=0$.

(3) $\delta$ is sub-modular.

(4) Setting, for $A \subseteq B \in \mathcal{C}$, the relation $A \leq B \Longleftrightarrow \delta(A) \leq \delta\left(A^{\prime}\right)$ for all $A \subseteq A^{\prime} \subseteq B$, makes $(C, \leq)$ into an amalgamation class.

(5) If $A \leq B_{1}, A \leq B_{2}, B_{i} \in \mathcal{C}$ then there is at most one structure $D \in \mathcal{C}$ (up to isomorphism) which is an $\leq$-amalgam for $B_{1}$ with $B_{2}$ over $A$, satisfying $\delta\left(D / B_{2}\right)=\delta\left(B_{1}\right)$. Moreover, if there is no $A \subsetneq B_{1}^{\prime} \subseteq B_{1}$ such that $B_{1}^{\prime} \hookrightarrow B_{2}$ then such $D$ - the free amalgam of $B_{1}$ with $B_{2}$ over $A$ - does exist.

(6) The Fraïsé limit of $(\mathcal{C}, \leq)$ is a saturated model of its own theory.

By Corollary 3.4.5 of [Wag94] stable pre-dimensioned classes produce $\omega$ stable theories (if $|\mathcal{C}|=\aleph_{0}$ ). In those terms, stable pre-dimensioned classes produce structures which are flat (in the sense of Hrushovski's, as quoted above) over the data, in the sense that any group configuration in the Frässé limit of $\mathcal{C}$ arises from one in $\mathcal{C}$. More precisely, if $\mathcal{U}$ is the Fraïssé limit of $\mathcal{C}$ and $Q \subseteq \mathcal{U}$ is an algebraic quadrangle, there is $C \in \mathcal{C}, R \leq C$ and an embedding $f: C \hookrightarrow \mathcal{U}$ satisfying $f(R)=Q$.

More generally, if we define, in the natural way, for a stable pre-dimensioned class $\mathcal{C}$, replacing the notion of acl with that of cl-closure, we can readily define $\delta$-quadrangles in $\mathcal{C}$, or whether $\mathcal{C}$ is $n$-ample, non $(n+1)$-ample (what about the interpretability of an infinite field), properties which will all be carried out to the Fraïssé limit of $\mathcal{C}$.

The considerable amount of work invested over the years around Hrushovski's amalgamation constructions improved our understanding of their mechanism and our mastery of their manipulation; it changed significantly our view on the diversity of possible phenomena in strongly minimal theories, but it has not taught us how stretch these constructions further. Of all the 
many variants of these constructions (ultimately producing structures of finite rank) that I know, only the one of [Bau96] uses a pre-dimension function that is not an immediate derivations of the ones introduced in Hrushovski's original works. So to my taste, the true challenge in this field of constructions, is to find new stable pre-dimensioned classes. Ones which are $n$-ample non $(n+1)$-ample for large (i.e. $>1$ !! $) n$, ones in which algebraic quadrangles do not arise from known ranked groups, ones with new pre dimension functions. And what about the collapse? I believe it will take care of itself.

3.2. The collapse. The problems in this subsection are of a less general nature than the ones in the previous subsection, but since they are more closely related to the discussion of the previous section, it may be appropriate to present them here.

To the best of my knowledge, Cherlin's question (quoted in [Hru92], but not appearing in any of Cherlin's own papers) concerning the existence of a maximal minimal theories has not been completely settled in the strongly minimal case. Of course, the work of [Hru92] gives a negative answer for strongly minimal theories with DMP, or even to ones with a strongly minimal expansion with DMP. It is therefore quite natural to ask:

Problem 3.13. Does every strongly minimal set have a strongly minimal expansion with DMP?

It seems plausible, that if we restrict ourselves to rank preserving expansions the answer to the question is negative (an adaptation of the construction of [HH06b] to the example of $\S 3$ in [Has07] could do the trick) but in its full generality the question seems to me completely open.

Another, possibly of more interest, approach to Cherlin's question could be:

Problem 3.14. Let $T_{1}, T_{2}$ be strongly minimal theories (with $Q E$ ) in (countable) disjoint languages. Is there a strongly minimal completion of $T_{1}^{\forall} \cup T_{2}^{\forall}$.

Of course, a positive answer to Problem 3.13 will also answer positively this last question, but it may not be the easiest way of answering it. As already mentioned earlier, there is no problem construction an $\omega$-stable completion $T_{\infty} \supseteq T_{1}^{\forall} \cup T_{2}^{\forall}$ of rank $\omega$, and the natural question is whether this theory can be collapsed. As pointed out in Subsection 2.3, in that generality $T_{\infty}$ need not have definability of strong minimality, though it will have a weaker version thereof, namely for a strongly minimal $\varphi(x, b)$ there is $n \in \mathbb{N}$ and $\theta \in \operatorname{tp}(b)$ such that $\operatorname{MR}(\varphi(x, b l))=1$ and its multiplicity at most $n$, for all $b^{\prime}=\theta$. This in itself suffices for the construction of envelopes (since $T_{\infty}$ will still not have obstructions), but when trying to construct pseudoenvelopes things become more delicate. Apparently, a closer look at pseudo obstructions and the ways they can arise will be needed.

In all probability if the fusion of strongly minimal sets can be carried out without DMP, the same techniques could be adapted to complete the 
characterization, started in [Has07], of structures of finite Morley rank interpretable in strongly minimal structures, and in particular answer the following fairly natural question:

Problem 3.15. Is every uncountably categorical theory interpretable in a strongly minimal one? What if we require the interpretation to be rank preserving?

In fact, I don't know the answer to the above question even for almost strongly minimal theories.

\section{REFERENCES}

[Bal94] John T. Baldwin. An almost strongly minimal non-Desarguesian projective plane. Trans. Amer. Math. Soc., 342(2):695-711, 1994.

[Bal02] John T. Baldwin. Rank and homogeneous structures. In Tits buildings and the model theory of groups (Würzburg, 2000), volume 291 of London Math. Soc. Lecture Note Ser., pages 215-233. Cambridge Univ. Press, Cambridge, 2002.

[Bau96] Andreas Baudisch. A new uncountably categorical group. Trans. Amer. Math. Soc., 348(10):3889-3940, 1996.

[BH] John Baldwin and Kitty Holland. Constructing $\omega$-stable structures: Rank 2 fields.

[BHMPW06] Andreas Baudisch, Martin Hils, Amador Martin-Pizarro, and Frank Wagner. Die böse farbe. Available on http://igd.univ-lyon1.fr/ wagner/publ.html\#prep, 2006.

[BMPZ05a] Andreas Baudisch, Amador Martin-Pizarro, and Martin Ziegler. Red fields. Available on http://home.mathematik.unifreiburg.de/ziegler/Preprints.html, 2005.

[BMPZ05b] Andreas Baudisch, Amador Martin-Pizzaro, and Martin Ziegler. Fusion over vector spaces. Available on http://home.mathematik.unifreiburg.de/ziegler/Preprints.html, 2005.

[BP00] Andreas Baudisch and Anand Pillay. A free pseudospace. Journal of Symbolic Logic, 65(2):914-922, 2000.

[CH03] Gregory Cherlin and Ehud Hrushovski. Finite structures with few types, volume 152 of Annals of Mathematics Studies. Princeton University Press, Princeton, NJ, 2003.

[CHL85] Gregory Cherlin, Leo Harrington, and A.H. Lachlan. $\aleph_{0}$-categorical, $\aleph_{0^{-}}$ stable structures. Annals of Pure and Applied Logic, 28:103-135, 1985.

[CL86] Gregory Cherlin and Alistair Lachlan. Finitely Homogeneous Structures. Transactions of the American Mathematical Society, 296(2):815-850, 1986.

[EH95] David Evans and Ehud Hrushovski. The automorphism group of the combinatorial geometry of an algebraically closed field. J. of the London Mathematical Society, 52(2):209-225, 1995.

[Has04] Assaf Hasson. Collapsing structure and a theory of envelopes. Prepritn, 2004.

[Has07] Assaf Hasson. Interpreting structures of finite morley rank in strongly minimal sets. Annals of Pure and Applied Logic, 145(1):96-114, 2007.

[HH06a] Assaf Hasson and Martin Hils. Fusion over sub-languages. Journal of Symbolic Logic, 71(2):361-398, 2006.

[HH06b] Assaf Hasson and Ehud Hrushovski. DMP in strongly minimal structures. To Appear in J. Symbolic Logic, 2006. 
[HI03] E. Hrushovski and M. Itai. On model complete differential fields. Trans. Amer. Math. Soc., 355(11):4267-4296 (electronic), 2003.

[Hru92] Ehud Hrushovski. Strongly minimal expansions of algebraically closed fields. Israel Journal of Mathematics, 79:129-151, 1992.

[Hru93] Ehud Hrushovski. A new strongly minimal set. Annals of Pure and Applied Logic, 62:147-166, 1993.

[Hru97] E. Hrushovski. Simplicity and the lascar group, 1997.

[Hru98] Ehud Hrushovski. A stable $\aleph_{0}$-categorical pseudoplane. 1998.

[Kir06] Jonathan Kirby. Exponential and weierstrass equations. Available at http://www.maths.ox.ac.uk/ kirby/ewe271.pdf, 2006.

[Lac84] Alistair Lachlan. Countable stable structures which are homegeneous for a finite relational langauage. Isreal Journal of Mathematics, 49:69-153, 1984.

[Pil95] Anand Pillay. The geometry of forking and groups of finite Morley rank. The Journal of Symbolic Logic, 60(4):1251-1259, December 1995.

[Pi196] Anand Pillay. Geometric stability theory, volume 32 of Oxford Logic Guides. The Clarendon Press Oxford University Press, New York, 1996. Oxford Science Publications.

[Pil00] Anand Pillay. A note on CM-triviality and the geometry of forking. The Journal of Symbolic Logic, 65(1):475-479, March 2000.

[Poi87] Bruno Poizat. Groupes stables. Nur al-Mantiq Wal-Ma'Rifah [Light of Logic and Knowledge],. Bruno Poizat, Lyon, 1987.

[Poi99] Bruno Poizat. Le Carré de L'Egalité. Journal of Symbolic Logic, 64(3):13391355, 1999.

[Poi01] Bruno Poizat. L'égalite au cube. Journal of Symbolic Logic, 66:1647-1676, 2001.

[Poi02] Bruno Poizat. Amalgames de Hrushovski: une tentative de classification. In Tits buildings and the model theory of groups (Würzburg, 2000), volume 291 of London Math. Soc. Lecture Note Ser., pages 195-214. Cambridge Univ. Press, Cambridge, 2002.

[Wag94] Frank O. Wagner. Relational structures and dimensions. In Automorphisms of first-order structures, Oxford Sci. Publ., pages 153-180. Oxford Univ. Press, New York, 1994.

[Zie06] Martin Ziegler. Fusion of structures of finite morley rank. Available on http://home.mathematik.uni-freiburg.de/ziegler/Preprints.html, 2006.

[Zil93] Boris Zilber. Uncountably Categorical Theories, volume 117. Translations of Mathematical Monographs, American Mathematical Society, Providence, RI., 1993.

[Zil05] Boris Zilber. Analytic and pseudo-analytic structures. In Logic Colloquium 2000, volume 19 of Lect. Notes Log., pages 392-408. Assoc. Symbol. Logic, Urbana, IL, 2005.

* Mathematical Institute, Oxford University, Oxford, UK

E-mail address: hasson@maths.ox.ac.uk

URL: http://www.maths.ox.ac.uk/ hasson/ 